\title{
Intramolecular Spin Alignment in Photomagnetic Molecular Devices: A Theoretical Study
}

\author{
Ilaria Ciofini, ${ }^{*[a]}$ Philippe P. Lainé, ${ }^{[b]}$ Marta Zamboni, ${ }^{[a]}$ Claude A. Daul, ${ }^{[c]}$ \\ Valérie Marvaud, ${ }^{[\mathrm{d}]}$ and Carlo Adamo ${ }^{[\mathrm{a}]}$
}

\begin{abstract}
Ground- and excited-state magnetic properties of recently characterized $\pi$-conjugated photomagnetic organic molecules are analyzed by the means of density functional theory (DFT). The systems under investigation are made up of an anthracene (An) unit primarily acting as a photosensitizer $(\mathrm{P})$, one or two iminonitroxyl (IN) or oxoverdazyl (OV) stable organic radical(s) as the dangling spin carrier(s) (SC), and intervening phenylene connector(s) (B). The magnetic behavior of these multicomponent systems, represented here by the HeisenbergDirac magnetic exchange coupling $(J)$, as well as the EPR observables ( $g$ tensors and isotropic $A$ values), are accurately modeled and rationalized by using our DFT approach. As the capa-
\end{abstract}

bility to quantitatively assess intramolecular exchange coupling $J$ in the excited state makes it possible to undertake rational optimization of photomagnetic systems, DFT was subsequently used to model new compounds exhibiting different connection schemes for their functional components $(\mathrm{P}, \mathrm{B}, \mathrm{SC})$. We show in the present work that it is worthwhile considering the triplet state of anthracene, that is, $\mathrm{P}$ when promoted in its lowest photoexcited state, as a full magnetic site in the same capacity as the remote SCs.

Keywords: density functional calculations - magnetic properties - molecular spintronics $\cdot$ photomagnetic molecular devices $\cdot$ radicals
This framework allows us to accurately account for the interplay between transient $\left({ }^{3} \mathrm{An}\right)$ and persistent (IN, OV) spin carriers, which magnetically couple according to a sole polarization mechanism essentially supported by phenyl connector(s). From our theoretical investigations of photoinduced spin alignment, some general rules are proposed and validated. Relying on the analysis of spin-density maps, they allow us to predict the magnetic behavior of purely organic magnets in both the ground and the excited states. Finally, the notion of photomagnetic molecular devices (PMMDs) is derived and potential application towards molecular spintronics disclosed.

\section{Introduction}

Light is a means of choice to probe and manipulate properties of molecules and magnetism is a proven and particularly well-suited property for information handling and storage.
Hence, photomagnetism has emerged as one of the most promising approach in nanosciences.

Photomagnetism of molecule-based materials is nowadays a mature research field. ${ }^{[1]}$ The bulk nature of these molecular materials is recognized to play a determining role in [a] Dr. I. Ciofini, M. Zamboni, Prof. Dr. C. Adamo

Laboratoire d'Électrochimie et

Chimie Analytique (CNRS UMR-7575)

École Nationale Supérieure de Chimie de Paris

11, rue Pierre et Marie Curie, 75231 Paris Cedex 05 (France)

Fax: (+33)144-276-750

E-mail: ilaria-ciofini@enscp.fr

[b] Dr. P. P. Lainé

Laboratoire de Chimie et

Biochimie Pharmacologiques et Toxicologiques (CNRS UMR-8601)

Université René Descartes

45, rue des Saints Pères, 75270 Paris Cedex 06 (France)

Fax: $(+33) 142-868-387$

E-mail: philippe.laine@univ-paris5.fr [c] Prof. Dr. C. A. Daul

Département de Chimie, Université de Fribourg Pérolles, 1700 Fribourg (Switzerland)

[d] Dr. V. Marvaud

Laboratoire de Chimie Inorganique et Matériaux Moléculaires (CNRS UMR-7071)

Université Pierre et Marie Curie

4, Place Jussieu, 75252 Paris Cedex 05 (France) 
both the successful achievement of their photomagnetic activity and the multifunctional integration they require as "smart materials". $[2,3]$ Thereby, the multifaceted nature of molecular object is often turned to good account by properly applying modern concepts of supramolecular chemistry, even though adjustments inherent to magnetism are sometimes advisable. ${ }^{[4]}$ As a matter of fact, much less work has been devoted to photomagnetism essentially relying on the sole molecular basis. ${ }^{[5-7]}$ Indeed, the more than two thousand years old Magnetism ${ }^{[8]}$ has only recently sprouted the branch of molecular magnetism. ${ }^{[9-13]}$ Meanwhile, the last decades have also witnessed tremendous advances in the conceptual rationalization of multifunctional integration at the (intra-)molecular level (including photoactivity) ${ }^{[14-17]}$ and in performances of spectroscopic methods with respect to both time and space resolution. ${ }^{[18-24]}$ Concomitantly, theoretical tools have shown major developments allowing to accurately account for behaviors of ever-increasing complexity. ${ }^{[25]}$ Hence, brought together, these progressions have made possible recent materialization and study of prototype photomagnetic organic and inorganic molecular compounds. ${ }^{[26]}$

In this context, with the aim to design new photoswitchable molecule-based magnetic devices, an increasing interest has risen towards systems that can change their overall spin multiplicity upon light excitation. One approach consists in transiently organizing the intramolecular spin alignment of the inner spin carriers (SCs) of properly designed photoactive paramagnetic entities. In these systems, the SCs are arranged about a diamagnetic center (core) to which they are all connected through bridging units. Basically, the working principle of such photomagnetic systems relies on the change of the magnetic status of the core from diamagnetic to paramagnetic ${ }^{[27]}$ upon absorption of a photon. The localized change may result from a redox process (e.g. oxidation following electron transfer, ET), a photophysical process (e.g. triplet-state formation) and in principle even a magnetic process (spin-crossover phenomenon). In the initial ground state, the SCs are normally loosely coupled one each other through the diamagnetic core, while in the photopromoted state, they are all strongly coupled to the same central magnetic site. As a result, peripheral persistent SCs are correlated (spin alignment) regardless of whether magnetic coupling with the activated core is ferro- or antiferromagnetic. Light activation of the core can be performed either 1 ) directly or 2) indirectly. In the former, case 1 , the closedshell core primarily functions as a photosensitizing chromophore $(\mathrm{P})$. Upon light absorption, $\mathrm{P}$ can either be simply promoted into its paramagnetic lowest excited state (Scheme 1a) or undergo an ET to one of the SC peripheral units (Scheme 1b) ${ }^{[6 a]}$ In the latter, case 2 , the core is activated by a photoactive remote site undergoing an ET or an electronic energy transfer (EnT; Scheme 1c). ${ }^{[28]}$

In the following, we only consider case 1, that is, the direct-light activation of the core, which therefore has primarily the function of a photosensitizer $(\mathrm{P})$. Depending on whether the light-triggered activation mode of this core $(\mathrm{P})$ is based on a purely photophysical process (Scheme 1a) or

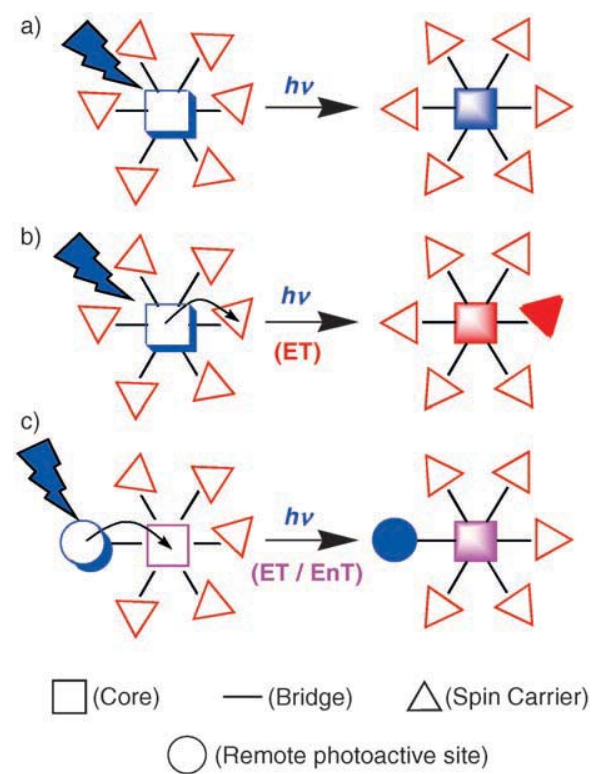

Scheme 1. Working principle of photoactivated high-spin molecules (three selected cases).

involves a redox process (Scheme $1 b$ ), the very nature of the activated $\mathrm{P}$ (as spin carrier) will be different. In both cases, however, ${ }^{*} \mathrm{P}$ effectively plays the role of a nexus core for the intramolecular spin alignment by virtue of its central location, and when activated.

Clearly, the larger the number of peripheral SCs (arbitrarily set to six SCs in the Scheme 1) the greater the photomagnetic effect is expected. In this respect, so-called highspin molecules are therefore potentially desirable as recently demonstrated by one of us, ${ }^{[6]}$ who devised photoswitchable octacyanomolybdate-based heptanuclear magnetic compounds. ${ }^{[6 a, 29]}$ Thus, both the photoactive $\mathrm{P}$ element and the persistent spin carriers (SCs) can be complexes of metal ions, usually from the d-block, from the first row for the SCs and from the second or third row for the P. The use of such inorganic building blocks is likely to favor a working mode that involves intramolecular ET (Scheme 1b). However, the $\mathrm{P}$ and the SCs can also be purely organic components, possibly encompassed in hybrid organic-inorganic assemblies. Actually, it is worth classifying the various compounds as a function of the nature of the bridging elements and related connection types. On the one hand, there are systems in which SCs and the photoactive nexus core $(\mathrm{P})$ are connected by coordination or sigma bonds. Examples of prototype systems of this kind are the fullerene-mononitroxide assemblies, ${ }^{[30]}$ the tetraphenylporphyrinatozinc(II) complex with $p$ pyridylnitronylnitroxide (ZnTPP-nitpy; in the solid phase $)^{[31]}$ and the above-mentioned octacyanomolybdatebased heptanuclear compounds. ${ }^{[6 a]}$ On the other hand, there are assemblies in which these units are $\pi$ conjugated, the coupling being expected to be the strongest for these sytems. Prototype systems of this second class are rather scarce and we believe are essentially represented by purely organic molecules designed by Teki, in Osaka. ${ }^{[2,33]}$ 
Thus, Teki and collaborators ${ }^{[32 b-d, g-j]}$ have recently synthesized and characterized an intriguing series of molecular systems (Figure 1a) containing two stable radicals as SCs, namely oxoverdazyl $(\mathrm{OV})^{[34]}$ or iminonitroxyl (IN; note in IUPAC nomenclature the use of aminoxyl instead of nitrox-

a)

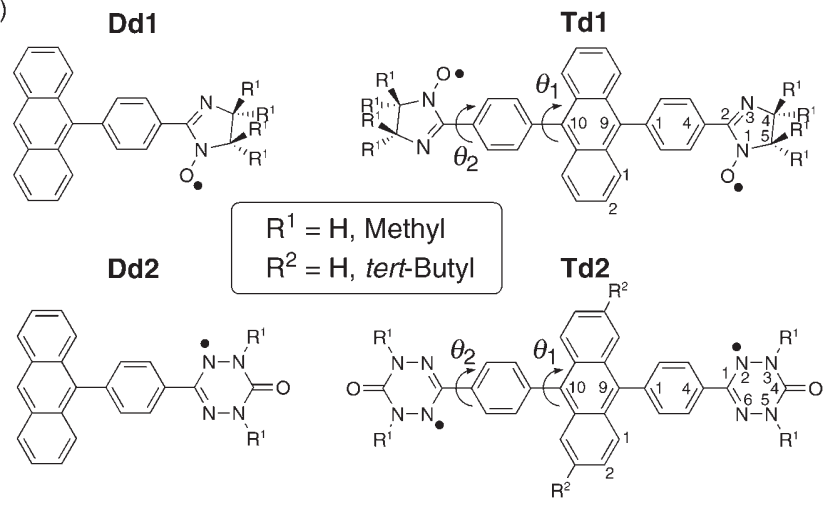

b)

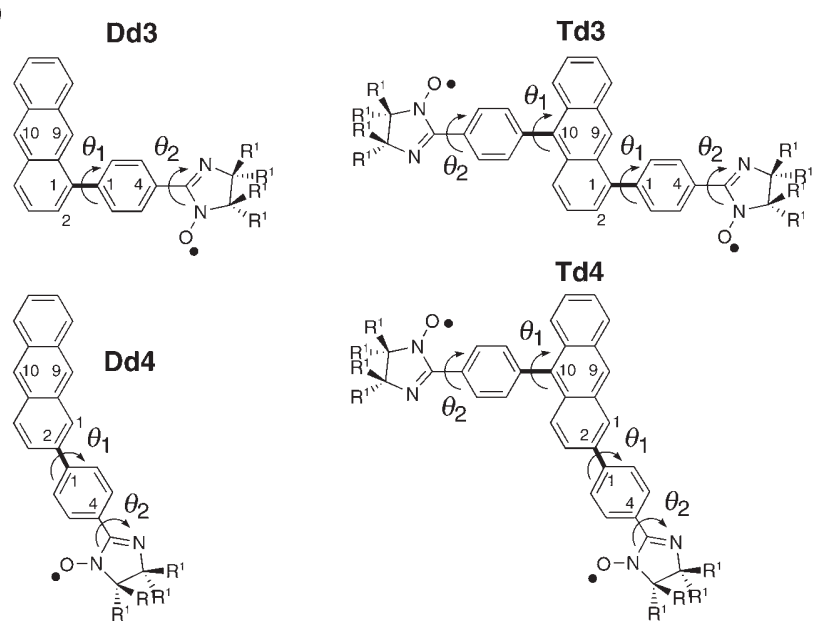

Figure 1. Schematic drawing and labeling schemes of various photomagnetic molecular systems studied. a) Experimentally characterized species. ${ }^{[32 b-d, g-j]}$ b) Alternative connection schemes.

$\mathrm{yl}$ is recommended) ${ }^{[35]}$ connected to an anthracene $(\mathrm{An})^{[36]}$ core-as the $\mathrm{P}$ unit-by $p$-phenylene (ph) bridging elements. They have investigated the coupling between the SCs both in the ground and in the excited states. Photoinduced spin alignment has been studied by carrying out involved spectroscopic experiments, including time-resolved electron-spin resonance. In principle, the working mode derived for these prototype photomagnetic systems should relate back to the one depicted in Scheme 1a. Indeed, in the ground state, the two SCs of the diradical systems (Td1 and Td2; Figure 1a) are weakly antiferro- magnetically coupled through the diamagnetic spin coupler made up of 9,10-diphenylanthracene [ph-An-ph]. Upon light-excitation of the assembly, An is promoted to its excited paramagnetic $(S=1)$ triplet state $(\mathrm{T})$, which couples ferromagnetically with the dangling stable radicals through intervening phenyls. A quintet state $(\mathrm{Qn} ; S=2)$ results from the intramolecular spin alignment in the excited state and was thus observed for the first time in a purely organic system. ${ }^{[32 j]}$ Similarly, Teki and collaborators also managed to demonstrate the formation of purely organic excited quartet $(S=3 / 2)$ state (Qr) in the case of affiliated monoradical species (Dd1 and Dd2; Figure 1a). Moreover, they have shown that in these open-shell organic molecules, intersystem crossing leading to the Qr and Qn thermally equilibrated excited (thexi) states is at one and the same time enhanced and selective. ${ }^{[32]}$

From a theoretical viewpoint, the same authors have performed preliminary ab-initio calculations, based on density functional theory (DFT), in order to clarify the mechanism of coupling in the excited state. ${ }^{[32 i]}$ In particular, by analyzing the spin-density (SD) patterns computed for the high-spin excited-state, they proposed the mechanism depicted in Scheme 2. The coupling of the two dangling radicals is explained in terms of "prevalence of SD delocalization over SD polarization onto part (namely the anthracene domain) of the *[ph-An-ph] photoexcited spin coupler, resulting in an overall polarization pattern favorable to ferromagnetic interaction of remote SCs". Thus, it was inferred that, in the excited state, spin delocalization mechanism within the anthracene moiety of the "spin coupler" overcomes the spin polarization effect, which prevails in the ground state.

We show in the present work that it is worthwhile alternatively considering triplet-state of anthracene as a full magnetic site in the same capacity as the two remote SCs, which couples with other site(s) according to the sole polarization mechanism essentially supported by phenyl connector(s). Thus, these appealing photomagnetic systems can also be viewed as prototype molecules for photoswitchable, purely organic, high-spin molecules working according to the principle depicted in Schemes 1a and 3.

In spite of the potential importance of (intramolecular) spin alignment upon photonic interactions in the main-
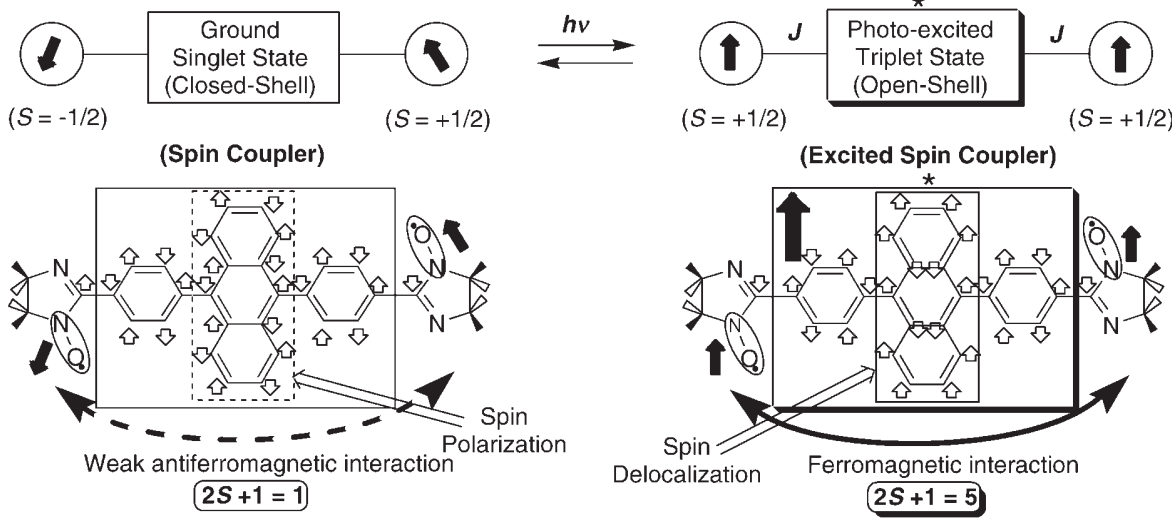

Scheme 2. Working principle of the herein studied photomagnetic systems according to Teki. ${ }^{[32]}$ 

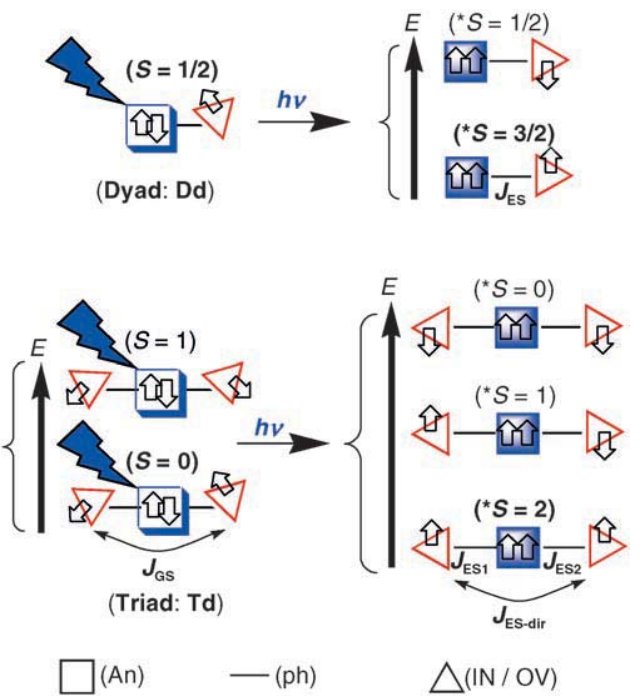

Scheme 3. Alternative working principle proposed in this work for the herein studied photomagnetic systems (see also Scheme 1a).

stream of advanced photoresponsive magnetic-moleculebased systems, theoretical research devoted to this phenomenon are rare. ${ }^{[32 i, 37]}$ In particular, determination of exchange coupling in the excited state has not been assessed yet. However, capability of determining the magnitude of intramolecular exchange couplings in the excited state is a necessary (key) step towards rational optimization of photomagnetic efficiency of molecular devices. As such quantities are not readily available from experiment, their theoretical assessment is mandatory.

In the present contribution, we used DFT to qualitatively and quantitatively evaluate the magnetic properties of the well-documented diradical species (Td1 and Td2, Figure 1a) and their affiliated mono-radicals molecules (Dd1 and Dd2) in order to model their excited state behavior. Starting from structural and spin-density analyses, the coupling between the spin carriers and the role of the spin coupler in both the ground and excited states was investigated. In particular, using the broken symmetry (BS) approach, we were able to compute the magnetic coupling in both the ground and excited states, thus giving not only a qualitative but also a quantitative estimate of the exchange coupling constant in the excited state (ES; $J_{\mathrm{ES}}$, Scheme 3 ). The main EPR spectroscopic parameters ( $A_{\text {iso, }}$ electronic $g$ tensors) were also computed. The reliability of the computational procedure was therefore confirmed by the good agreement found with respect to the experimental data available for the existing reference systems. This theoretical approach was subsequently applied to investigate the photomagnetic behavior of other (new) mono- and diradical species (Dd3, Dd4, Td3, and Td4) showing alternative connection schemes for their functional components (Figure 1b).

Actually, the series of photoresponsive molecules devised and characterized by Teki yields an unique opportunity to assess the pertinence of defining supramolecular photomagnetism, being understood within the same context as that of the supramolecular photochemistry paradigm developed by Balzani for photochemical molecular devices (PMDs) ${ }^{[14]}$ that is, in the present case, assembly of components (polyads) to obtain photomagnetic molecular devices (PMMDs). Herein, we show that such an analytical grid indeed allows the accurate qualitative description of photomagnetic behaviors observed so far, in addition to providing the means for identifying proper model compounds to compute key intensive quantities, such as exchange coupling constants $(J)$ attached to both the ground and excited states of the two- (dyad) and three-component (triad) molecular systems. In doing so, a conceptual framework allowing accurate description of the interplay between transient and persistent magnetic sites is proposed together with criteria for unambiguously defining the basic notions of magnetic (super)sites, connecting elements and attached exchange-coupling pathways for PMMDs.

Guidelines for improving photomagnetic behaviors were finally derived and potential application of PMMDs towards molecular spintronics is disclosed.

\section{Computational Methods}

All calculations, except those of $g$ tensors, were performed in the gas phase by using the Gaussian 03 package. ${ }^{[38]}$ The hybrid PBE0 exchangecorrelation functional, ${ }^{[39]}$ casting $25 \%$ of Hartree-Fock exchange in the parent generalised gradient approximation (GGA) functional (i.e., Perdew-Burke-Ernzerhof; PBE) ${ }^{\left[{ }^{[0]}\right]}$ was used throughout for calculations of both structural and magnetic properties within unrestricted formalism. Structural optimizations and subsequent frequency calculations were performed by using the Dunning-Hay double-zeta valence basis set, ${ }^{[41]}$ hereafter referred to as LANL2 basis.

Larger basis sets were used to compute magnetic properties such as the isotropic exchange coupling constant $(J)$ and the isotropic, Fermi, hyperfine interaction $\left(A_{\text {iso }}\right)$. For such calculations, a Pople double-zeta split valence basis $(6-31 \mathrm{G})^{[42]}$ was used for all atoms belonging to the central diphenyl-anthracene unit, while all other non-hydrogen atoms were described by the same basis enhanced by one $d$ polarization function and a diffuse function $(6-31+G(d)) \cdot{ }^{[42]}$ This ensemble of basis sets will be referred to as Pople mix $_{\text {in }}$ the following.

To compare with the experimental results, the magnetic exchange interaction between the radical units was interpreted using the HeisenbergDirac-Van Vleck (HDVV) spin Hamiltonian [Eq. (1)]. ${ }^{[4-45]}$

$H_{\mathrm{HD}}=-2 \sum_{i, j} J_{i j} \vec{S}_{i} \vec{S}_{j}$

Using this notation, negative $J$ values correspond to an antiferromagnetic interaction, while positive ones are related to a ferromagnetic interaction. To compute the $J$ values, the broken-symmetry (BS) approach was applied. ${ }^{[46-48]}$ In particular, $J$ was computed from Equation (2), in which $\left\langle S^{2}\right\rangle_{\mathrm{HS}}$ and $\left\langle S^{2}\right\rangle_{\mathrm{BS}}$ are the expected value of $S^{2}$ computed for the high-spin (HS) and broken-symmetry states, respectively.

$J_{i j}=-\frac{\left(E_{\mathrm{HS}}-E_{\mathrm{BS}}\right)}{\left\langle S^{2}\right\rangle_{\mathrm{HS}}-\left\langle S^{2}\right\rangle_{\mathrm{BS}}}$

The $J$ values, reported in the Tables as $J / k_{B}$, are expressed in $\mathrm{K}$ units to facilitate comparison with available experimental data. Isotropic hyper- 
fine interaction values ( $A_{\text {iso }}$, Fermi contact terms), were computed from Equation (3) ${ }^{[49]}$ in which $\beta_{\mathrm{e}}, \beta_{\mathrm{N}}$ are the electron and nuclear magnetons, respectively, $g_{e}, g_{\mathrm{N}}$ are the corresponding magnetogyric ratios, $h$ the Planck constant, $\delta(r)$ is a Dirac delta operator and $P_{\mu \nu}^{\alpha-\beta}$ is the spin-density matrix.

$a_{\mathrm{N}}=\frac{4 \pi}{3 h\left\langle S_{\mathrm{z}}\right\rangle} g_{\mathrm{e}} \beta_{\mathrm{e}} g_{\mathrm{N}} \beta_{\mathrm{N}} \sum_{\mu, v} P_{\mu \nu}^{\alpha-\beta}\left\langle\varphi_{\mu}\left|\delta\left(r_{\mathrm{kN}}\right)\right| \varphi_{\nu}\right\rangle$

Finally, $g$ tensors were computed by using the ORCA program ${ }^{[50]}$ and a 6-31G(d) basis for all atoms. The $g$ tensors are given as deviations $(\Delta g)$ with respect to the free-electron value $\left(g_{\mathrm{e}}=2.002319\right) . \Delta g$ is expressed in ppm. Considering a Pauli Hamiltonian up to $O(\alpha)^{2}$, there are three main contributions to $g$ shifts [Eq. (4)] ${ }^{[51]}$ in which the $\Delta g^{\mathrm{RMC}}$ and $\Delta g^{\mathrm{RMC}}$ contributions are first-order terms taking into account relativistic mass (RMC) and gauge (GC) corrections while the last term, $\Delta g^{\mathrm{RMC}}$, is a second-order contribution to the $g$-shifts arising from the coupling of the orbital zeeman (OZ) and the spin-orbit coupling (SOC) operators. This term can be expressed as Equation (5).

$\Delta g=\Delta g^{\mathrm{RMC}}+\Delta g^{\mathrm{RMC}}+\Delta g^{\mathrm{RMC}}$

$\Delta g_{\mathrm{kl}}^{\mathrm{SO} / \mathrm{OL}}=\sum_{\mu, v} \frac{\partial P_{\mu \nu}^{\alpha-\beta}}{\partial B_{k}}\left\langle\varphi_{\mu}\left|h_{v}^{\mathrm{SOC}}\right| \varphi_{\nu}\right\rangle$

In Equation (5), $h^{\mathrm{sOC}}$ is the spatial part of the effective one-electron spinorbit coupling operator and $B_{k}$ the $k$ component of the external magnetic field. In principle, the SOC is composed of one- and two-electron parts; however, it can be approximated by using a one-electron operator. The complexity of the exact calculation is then reduced accordingly. In this approach, the simplest approximation, based on the use of adjusted effective nuclear charges (hereafter Koseki), was applied. In this case, the one-electron approximate SOC operator simply reads as Equation (6), in which $\vec{l}_{\mathrm{A}}(i)$ is the angular momentum operator of the $i$-th electron relative to the nucleus A and $\vec{s}(i)$ its spin-operator. The function $\xi\left(r_{i, A}\right)$ is defined in Equation (7), ${ }^{[52]}$ in which $Z_{\text {eff }}^{\mathrm{A}}$ is the effective nuclear charge of atom $\mathrm{A}$ at position $\vec{R}_{\mathrm{A}}$.

$\hat{H}_{\mathrm{SOC}}=\sum_{\mathrm{A}, i} \zeta\left(r_{i, \mathrm{~A}}\right) \vec{l}_{\mathrm{A}}(i) \overrightarrow{\mathrm{s}}(i)$

$\zeta\left(r_{i, \mathrm{~A}}\right)=\frac{\alpha^{2}}{2} \frac{Z_{\mathrm{eff}}^{\mathrm{A}}}{\left|\vec{r}_{i}-\vec{R}_{\mathrm{A}}\right|^{3}}$

No attempts were made to compute zero-field splitting parameters, since it is known that spin-spin contributions to $D$, not included in the present calculations, are dominant with respect to the SOC terms for triplet states of linear polyacenes. ${ }^{[53]}$ Besides, we are aware that such terms have been recently included in the Orca package. ${ }^{[54]}$

In the following, all excited states will be suffixed with *. Finally, when atomic spin population (ASP) is discussed, we always refer to Mulliken spin population.

\section{Results and Discussion}

In the following, the properties of existing systems (Dd1, Dd2, Td1 and Td2; Figure 1a) will be first analyzed from structur- al, electronic, and magnetic viewpoints, allowing the validation of the theoretical approach against available experimental data. Then, theses issues will be discussed and rationalized in the framework of supramolecular photomagnetism, leading to the definition of photomagnetic molecular devices (PMMDs). On these bases, structural and magnetic behaviors of four new iminonitroxyl derivatives (Dd3, Dd4, Td 4 and Td4; Figure 1b) will be finally investigated.

Validation of the theoretical approach against available experimental data-structural insights into ground and excited states for Td1 and Td2: Since intramolecular structural features, and especially conformation, play a pivotal role in closely coupled and potentially fully $\pi$-conjugated, compact, systems, ${ }^{[55-57]}$ structural optimizations have to be done carefully.

Schematic representations and numbering schemes of systems Td1, Td2, Dd1, and Dd 2 are given in Figure 1a. In all calculations concerning iminonitroxide derivatives (Td1 and Dd1), methyl substituents $\left(\mathrm{R}^{1}\right)$ were replaced with hydrogen atoms. This simplification is widely applied ${ }^{[58,59]}$ as it does not significantly affect either the electronic or the structural overall properties of the systems. No such structural simplification was applied when modeling verdazyl derivatives Td2 and Dd2. In the case of Td2, however, we have checked that removal of tert-butyl substituents ${ }^{[32 \mathrm{~g}]}$ grafted on the anthracene core of molecules actually investigated at the experimental level has no effect on computational issues (see Table SI-II in Supporting Information).

The computed structural parameters are collected in Tables 1 and 2 for iminonitroxide (Td1 and Dd1) and oxoverdazyl (Td2 and Dd2) derivatives, respectively, together with experimental data available for similar systems.

In the ground state, structural features of the spin carriers of both the mono- (Dd1 and Dd2) and the diradical (Td1 and Td2) species are found to be practically insensitive to the overall spin state, thus confirming the intrinsic sturdiness of the IN and OV radicals. In all cases, the radical moieties are found to be planar, although no symmetry constraints were applied, yielding a pseudo- $C_{s}$ symmetry in the case of the IN moieties and retaining a pseudo- $C_{2 v}$ symmetry for the OV subunits.

When analyzing the iminonitroxide derivative Dd1 (Table 1$)$, we note a shorter $\mathrm{C}-\mathrm{N}$ imino bond $\left(d\left(\mathrm{C}^{\mathrm{IN}}-\right.\right.$

Table 1. Selected structural parameters computed for 9,10-diphenylanthracene (ph-An-ph) as well as iminonitroxide (IN) derivatives Td1 and Dd1 in the ground state (Td1, Dd1) and in the excited state (Td1*, Dd 1*) as a function of the overall spin state. Available X-ray data for similar systems are given for comparison purposes. Distances in $\AA$, angles in degrees. For labeling scheme refer to Figure $1 \mathrm{a}$.

\begin{tabular}{|c|c|c|c|c|c|c|c|c|}
\hline & $\begin{array}{l}\text { ph-An-ph } \\
S=0\end{array}$ & $\begin{array}{l}\text { ph-An-ph* } \\
S=1\end{array}$ & $\begin{array}{l}\text { Td1 } \\
\left(\mathrm{BS} ; m_{S}=0\right)\end{array}$ & $\begin{array}{l}\text { Td1 } \\
S=1\end{array}$ & $\begin{array}{l}\text { Td1* } \\
S=2\end{array}$ & $\begin{array}{l}\text { Dd1 } \\
S=1 / 2\end{array}$ & $\begin{array}{l}\text { Dd1* } \\
S=3 / 2\end{array}$ & $\begin{array}{l}\text { IN }\left(\operatorname{exptl}^{[\mathrm{a}]}\right) \\
S=1 / 2\end{array}$ \\
\hline$d \mathrm{C} 9^{\mathrm{An}}-\mathrm{C} 1^{\mathrm{Ph}}$ & 1.494 & 1.485 & 1.479 & 1.479 & 1.4790 & 1.492 & 1.476 & \\
\hline$d \mathrm{C} 4^{\mathrm{Ph}}-\mathrm{C} 2^{\mathrm{IN}}$ & & & 1.471 & 1.471 & 1.4677 & 1.471 & 1.467 & $1.472 / 1.476$ \\
\hline$d \mathrm{C} 2^{\mathrm{IN}}-\mathrm{N} 1$ & & & 1.419 & 1.419 & 1.418 & 1.419 & 1.418 & $1.380 / 1.369$ \\
\hline$d \mathrm{C} 2^{\mathrm{IN}}-\mathrm{N} 3$ & & & 1.311 & 1.311 & 1.313 & 1.311 & 1.313 & $1.279 / 1.243$ \\
\hline$\theta_{1}$ & 75.4 & 65.5 & 73.5 & 73.5 & 59.1 & 71.2 & 56.4 & \\
\hline$\theta_{2}$ & & & 1.1 & 1.1 & 1.2 & 2.4 & 1.1 & $7.0 / 2.2$ \\
\hline
\end{tabular}

[a] From X-ray structure of 1,1-dimethyl-2,5-bis-(iminonitroxyl-phenyl)-3,4-diphenylsilole (TPSIN). ${ }^{\text {[3a] }}$ 
$\left.\mathrm{N} 3^{\mathrm{IN}}\right)=1.311 \AA$ ) and a longer nitroxide-related $\mathrm{C}-\mathrm{N}$ bond $\left(d\left(C 2^{\mathrm{IN}}-\mathrm{N} 1^{\mathrm{IN}}\right)=1.419 \AA\right)$, in agreement with crystallographic data ${ }^{[33 a]}$ and previous calculations ${ }^{[59]}$ on systems containing IN units. These bond lengths are overestimated by approximately $0.045 \AA$ with respect to the experimental X-ray structure available for an analogous phenyl-substituted iminonitroxide fragment (1.279/1.243 and 1.380/1.369 A, respectively). ${ }^{[33 a]}$ The nitrogen-oxygen distance $\left(d\left(\mathrm{~N} 1^{\mathrm{IN}}-\mathrm{O}^{\mathrm{IN}}\right)=\right.$ $1.305 \AA)$ is also slightly overestimated with respect to experimental data (1.268/1.270 A, Table SI-I in Supporting Information). ${ }^{[33 a]}$ Both the intermolecular stacking and the presence of solvent, neglected in the current calculations, can account for the difference between computed and experimental data. Indeed, a better agreement with X-ray data is observed for $d\left(\mathrm{C}^{\mathrm{IN}}-\mathrm{N} 3^{\mathrm{IN}}\right)$ and $d\left(\mathrm{C}^{\mathrm{IN}}-\mathrm{N} 1^{\mathrm{IN}}\right)$ distances, the largest deviation being of $0.01 \AA$ (refer to Table SI-I). Similarly, bond angles, including $a\left(\mathrm{~N} 1-\mathrm{C} 2{ }^{\mathrm{IN}}-\mathrm{N} 3\right)$, are in very good agreement with experimental data (largest deviation $\approx 1^{\circ}$, Table SI-I).

Regarding the verdazyl derivatives, there is a good agreement with both X-ray data ${ }^{[60]}$ and previous calculations ${ }^{[61]}$ on systems containing the OV unit. In particular, when comparing calculated internal structure parameters of Dd 2 to experimental data ${ }^{[60]}$ (Table 2 and Table SI-II), maximum deviations for bond lengths and angles (including $a\left(\mathrm{~N} 6-\mathrm{Cl}^{\mathrm{OV}}\right.$ N2)) are $0.03 \AA$ and $2.0^{\circ}$, respectively.

Concerning the ground state of the spin-coupling assembly (that is, [ph-An-ph]), the calculated bond lengths and angles are virtually the same regardless of whether diphenylanthracene is isolated or embedded within Td1 or Td2, except for dihedral angle $\theta_{1}$ between the plane of the phenyl spacer and that of the central ring of anthracene (Figure 1a). In the case of the IN derivative (Td1), the system is computed to be slightly more flat by about $2^{\circ}$ (Table 1 ) than isolated diphenylanthracene. For the verdazyl derivative (Td2, Table 2), a flattening of the structure by $4^{\circ}$ is observed only in the case of the triplet state $(S=1)$ corresponding to an energy disadvantaged, ferromagnetic coupling of spin momenta of the remote SCs (see below). Effects of the same Dd1 and Dd2. This moderate conformational difference is most likely of electronic origin and related to $\pi$ conjugation with the dangling radical(s) $\left(\theta_{2} \leq 3^{\circ}\right)$. Nevertheless, the small amplitude of the flattening (about $\theta_{1}$ ) upon SC appending together with the fact that the internal geometric features of order of magnitude are also found for monoradical species

both the SCs and the spin coupler units are only scarcely modified, suggest that each component basically retains its structural integrity within the molecular assemblies.

Upon light-excitation, the only remarkable structural change in the considered systems is the planarization ${ }^{[62]}$ of the diphenylanthracene assemblies revealed by the computed values of $\theta_{1}$, which are getting noticeably smaller in the excited state than in the ground state (Tables 1 and 2). Again, even if such a type of structural relaxation is also computed for the parent diphenylanthracene upon $S_{0}$ to $T_{1}$ excitation $\left(\Delta \theta_{1}=10^{\circ}\right)$, the amplitude of the planarization is clearly enhanced by the presence of the dangling SCs. Indeed, on going from the ground to the excited state, a planarization about $\theta_{1}$ of $14.4^{\circ}$ and $17.7^{\circ}$ is obtained for $\mathbf{T d} 1^{*}$ and Td $2^{*}$ and of $14.8^{\circ}$ and $16.8^{\circ}$ for Dd $1^{*}$ and Dd $2^{*}$, respectively.

Spin density patterns and magnetic properties of Td1 and Td2-ground state properties (Td functional model): To rationalize the experimental magnetic behavior of Td1 and Td2, a single $J\left(J_{\mathrm{GS}} ; \mathrm{GS}=\right.$ ground state) parameter Dirac phenomenological Hamiltonian was used, in which $J_{\mathrm{GS}}$ describes the coupling between the spin carriers in the ground state (Scheme 3). Experimentally, it has been found that pairs of IN (Td1) and OV (Td2) SCs are weakly and antiferromagnetically interacting in the ground state. From the temperature dependence of the EPR signals, exchange coupling constants $\left(J_{\mathrm{GS}} / k_{\mathrm{B}}\right)$ could be determined: $-5.8 \mathrm{~K}$ for Td ${ }^{[32 \mathrm{~h}]}$ and $-3 \mathrm{~K}$ for Td2. ${ }^{[32 \mathrm{~g}]}$

The calculations reproduce correctly the relative energies of the different spin states for both Td1 and Td2. The antiferromagnetic state, here represented by the broken-symmetry solution, is predicted to be the most stable (see below).

In the case of the iminonitroxide triad (Td1) in its antiferromagnetic (ground) state, two different conformers of the diradical were analyzed, namely the trans and the cis conformers with respect to peripheral nitroxide moieties $(\mathrm{N}-$ $\mathrm{O})$. Both of the isomers correspond to minima on the potential energy surface. The trans form being more stable by only $0.9 \mathrm{~kJ} \mathrm{~mol}^{-1}$. Hence, we can state that the two forms should coexist although the barrier for interconversion should be higher (as in the case of biphenyl derivatives ${ }^{[63]}$ ).

To quantify the exchange coupling constants within Td1 and Td2, the broken-symmetry (BS) approach and the Pople $_{\text {mix }}$ basis set were used, according to the procedure deTable 2. Selected structural parameters computed for oxoverdazyl (OV) derivatives Td2 and Dd 2 in the ground state (Td2, Dd2) and in the excited state $\left(\right.$ Td2 $*$, Dd $\left.^{*}\right)$ as a function of the overall spin state. Available X-ray data for similar systems are given for comparison purposes. Distances in $\AA$, angles in degrees. For labeling scheme refer to Figure 1a.

\begin{tabular}{lcccccl}
\hline & Td2 $\left(\mathrm{BS} ; m_{S}=0\right)$ & $\mathbf{T d 2} S=1$ & $\mathbf{T d 2}^{*} S=2$ & Dd2 $S=1 / 2$ & Dd2 $^{*} S=3 / 2$ & OV $($ exptl) $S=1 / 2$ \\
\hline$d \mathrm{C}^{\mathrm{An}}-\mathrm{C} 1^{\mathrm{Ph}}$ & 1.493 & 1.492 & 1.478 & 1.493 & 1.476 & \\
$d \mathrm{C} 4^{\mathrm{Ph}}-\mathrm{C} 1^{\mathrm{OV}}$ & 1.477 & 1.477 & 1.473 & 1.477 & 1.473 & $1.477(2)^{[\mathrm{a}]}$ \\
$d \mathrm{C}^{\mathrm{OV}}-\mathrm{N} 2$ & 1.354 & 1.354 & 1.355 & 1.354 & 1.355 & $1.321(4) / 1.323(4)^{[\mathrm{b}]}$ \\
$d \mathrm{~N} 2-\mathrm{N} 3$ & 1.379 & 1.380 & 1.379 & 1.379 & 1.379 & $1.365(3) / 1.358(3)^{[\mathrm{b}]}$ \\
$\theta_{1}$ & 76.0 & 71.1 & 58.3 & 72.7 & 55.9 & \\
$\theta_{2}$ & 0.8 & 0.6 & 0.6 & 0.4 & 0.9 & \\
\hline
\end{tabular}

[a] X-ray structure of 1,3,5-triphenyl-6-oxoverdazyl from reference [60c]. [b] X-ray structure of 1,5-dimethyl-3(2-pyridyl)-6-oxoverdazyl radical from reference [60a]. scribed in the Computational Methods section.

The computed $J_{\mathrm{GS}} / k_{\mathrm{B}}$ values, reported in Table 3 , are in good agreement with the experimental data even though slightly underestimated, that is, $-0.2 \mathrm{~K}$ for Td1 (trans isomer) and $-0.1 \mathrm{~K}$ for $\mathbf{T d} 2$ were computed instead of $-5.8 \mathrm{~K}$ and $-3 \mathrm{~K}$, respectively, that was measured. ${ }^{[64]}$ 
Table 3. Computed intramolecular exchange coupling constants for Td1 and Td $2\left(J_{\mathrm{GS}} / k_{B}\right.$, in $\left.\mathrm{K}\right)$ in the ground state and for Dd 1* and Dd $2 *\left(J_{\mathrm{ES}} /\right.$ $k_{B}$, in $\left.\mathrm{K}\right)$ in the excited state. Negative $J$ values imply antiferromagnetic coupling.

\begin{tabular}{lllll}
\hline & Td1 & Td2 & Dd1* & Dd 2* \\
\hline$J_{\mathrm{GS}} / k_{\mathrm{B}}($ computed $)$ & $-0.2^{[\mathrm{b}]} /-5.4^{[\mathrm{c}]}$ & -0.1 & & \\
$J_{\mathrm{GS}} / k_{\mathrm{B}}($ exptl) & -5.8 & -3.0 & & \\
$J_{\mathrm{ES}} / k_{\mathrm{B}}$ & & & +127 & +177 \\
\hline
\end{tabular}

[a] From reference [32h] for Td1 and reference [32g] for Td2. [b] For Td1 with trans nitronyl groups. [c] For Td1 with cis nitronyl groups.

However, it should be borne in mind that evaluation of $J$ values was made by using the BS approach, which relies on a projection technique usually yielding larger errors both in absolute and relative values than post-HF methods. ${ }^{[65]}$ Therefore, the degree of agreement with experimental data reported here is considered to be within the precision range of the computational procedure applied. Most importantly, given that magnetic coupling strongly depends on structural features, we can reasonably state that optimized structures obtained here also correctly characterize the ground state of the molecules.

From the viewpoint of electronic structures, the unpaired electrons (here evaluated by analyzing the Mulliken atomic spin population, ASP) are virtually fully localized on the dangling radicals (IN or OV units): the overall spin density localized on the anthracene core is negligible $(<1 \%)$ for both ferromagnetic and antiferromagnetic states. Indeed, a small fraction of spin density with opposite sign $(\approx 1 \%)$ is found to be localized on the phenyl B-subunits, even though these moieties are strongly conjugated with the SCs $\left(\theta_{2}<\right.$ $\left.3^{\circ}\right)$. This issue is consistent with the structural picture computed for the ground state, confirming that each of the radical units basically retains its identity within the polyad systems.

Additional information on the coupling mechanism can be inferred from the analysis of spin-density maps, as already proposed by Teki and others. ${ }^{[32,66,67]}$ It is worth noting that a spin polarization pattern is present on the phenyl bridging subunits and also on the diamagnetic anthracene core, as revealed by the spin-density maps computed for Dd 1 and Dd 2 (Figure 2). Nevertheless, if the spin polarization mechanism yields larger spin-density patterns on the phenyl (with an overall negative spin density of $0.1 \mathrm{e}^{-}$on this subunit), the much smaller fraction of spin density computed on each atom of the anthracene unit as well as the negligible overall spin density localized on it, comply with the very weak coupling found between the two dangling radicals of Td1 and Td2. Actually, the closed-shell anthracene unit exhibits a poor superexchange pathway for magnetic interaction, thus accounting for the small values computed and measured for $J_{\mathrm{GS}} / k_{\mathrm{B}}$.

Still, in an attempt to assess the coupling between the SCs within the diradical triad species, one can make good use of the spin-density patterns computed for the corresponding dyads, in line with the approach proposed by Teki. ${ }^{[32]} \mathrm{We}$ first concentrated on the more documented case of Dd1
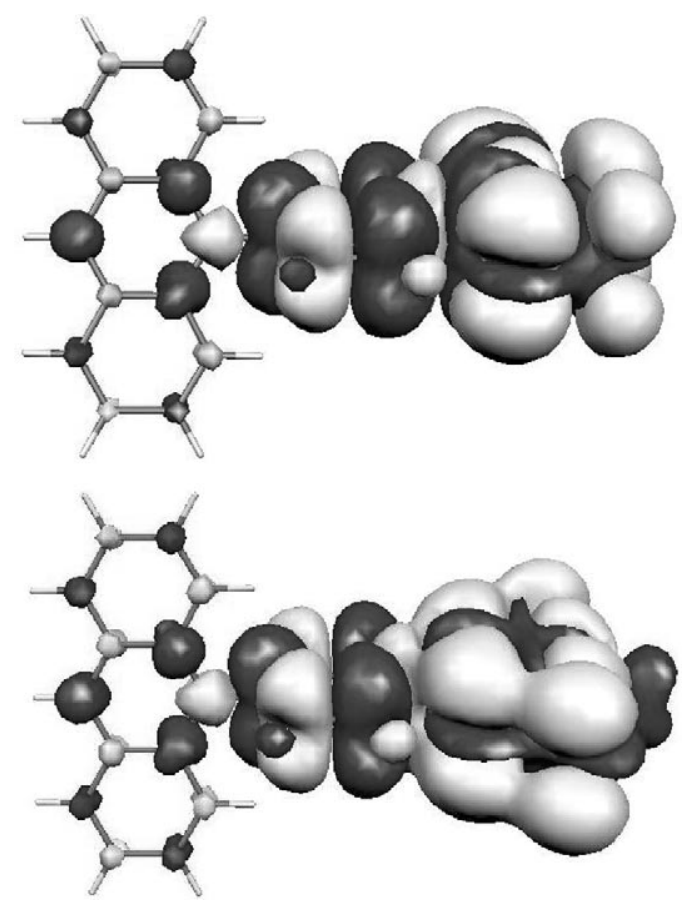

Figure 2. Spin-density patterns computed for Dd1 (top) and Dd2 (bottom). $S=1 / 2$; contour value $5 \times 10^{-5}$ a.u.

iminonitroxide derivative, since the same type of arguments holds for the verdazyl derivative. Spin density map of Dd1 (Figure 2) clearly reveals that appending of SC results in a polarization pattern of SD over the An core, which is rather low but sufficient to induce polarizations of opposite signs at positions 9 and 10. Therefore, in the case of the 9,10-disubstituted anthracene diradical (that is, Td1), the two SCs polarize in-phase the spin coupler (An) through the bridging phenyl connectors only if they are antiferromagnetically coupled. In contrast, for the case of a ferromagnetic alignment of the spin borne by remote SCs, the spin polarization patterns (characterized by sign alternation of spin densities on adjacent nuclei of the $\pi$-system network) originating from each of the two radicals are out-of-phase (that is, contributions of opposite sign on the same atoms; "destructive interference"), giving rise to a mismatch domain situated on the anthracene backbone, revealed by an overall lowering of local ASPs including at the key 9- and 10-positions (Figure 3$)$. As a result, the triplet state $(S=1)$ is higher in energy than the singlet state, and the latter is therefore the genuine ground state. Clearly the overall coupling will remain weak, since the spin density computed on the atoms of anthracene is small. The same reasoning based on the matching of spin polarization patterns originating from dangling radicals, in agreement with well-established $\pi$-topological rules, ${ }^{[68]}$ allows us to anticipate a ferromagnetic ground state for 9,2-disubstituted anthracene-based triad (hereafter denoted Td4), whereas an antiferromagnetic ground state is inferred for a 9,1-disubstituted triad isomer (hereafter denoted Td3). The validity of these inferences is discussed in greater detail below. 


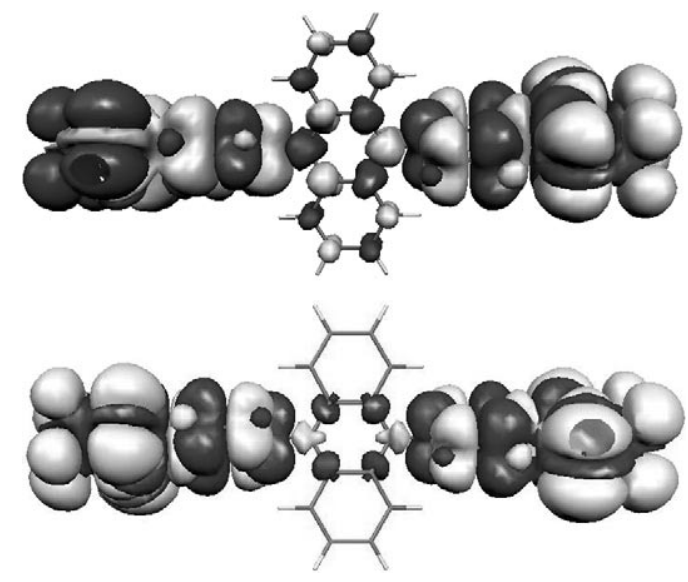

Figure 3. Spin-density patterns computed for the BS state $\left(m_{\mathrm{S}}=0\right.$; top $)$ and triplet state ( $S=1$; bottom) of $\mathbf{T d 1}$ (contour value $5 \times 10^{-5}$ a.u.).

Clearly, bridging phenyls are strongly involved in the spin-polarization mechanism as connectors. This active role of the phenyl rings essentially relies on the fact that they are roughly coplanar with the radical rings as evidenced by the $\theta_{2}$ tilt angles, which are systematically lower than $3^{\circ}$. At this point, relevance of the functional scheme hitherto used to account for the photomagnetic behavior of systems here considered can be questioned. These species can no longer be regarded as merely being made up of a diphenylanthracene as the photoresponsive spin coupler (even connector) and of the dangling radicals (IN or OV) as the SCs (see Scheme 2). ${ }^{[32]}$ In particular, phenyl groups can safely be considered as being the natural extension of radicals due to pronounced $\pi$ conjugation rather than being preferably associated to the anthracene core, to which they are loosely coupled as indicated by $\theta_{1}$ values ( $73^{\circ}$ on the average). Actually, there is room for the bridging connectors to get their own functional identity (see below). Accuracy of functional description is of pivotal importance to properly choose functional model species (i.e., dyads) to assess excited state magnetic behavior.

A more quantitative estimate of the spin delocalization can be derived from the analysis of the isotropic hyperfine coupling constants $\left(A_{\text {iso }}\right)$ computed for the mono- and diradical species. The calculated values, along with available experimental data, are gathered in Table 4.

These data unambiguously show that 1) the radicals are centered on the IN and $\mathrm{OV}$ backbones and 2) SD perturbation (that is, spin polarization) is spreading over the attached phenyl rings and even on the anthracene units on which some spin density is found, giving rise to significant $A_{\text {iso }}$ values for the $\mathrm{C}$ atoms at positions 9 and 10 (Figure 1 ). In addition, the good agreement with the experimental data obtained for the $A_{\text {iso }}$ values of the nitrogen nuclei of both IN and OV fragments further substantiates the relevance of our computational procedure. From the experimental data reported in Table 4, it is also clear that in the case of OV derivatives, the $A_{\text {iso }}$ value of the nitrogen nuclei at positions 2 and 3 strongly depends on the substituent linked to $\mathrm{C}^{\mathrm{OV}}$.

Spin-density patterns and magnetic properties of Td1 and Td2-excited state properties (Dd* functional model): Upon light-excitation of Td1 and Td2, a triplet excited state essentially located on the anthracene moiety is formed, giving rise to a metastable excited quintet state (Td $\mathbf{1}^{*}$ and Td $2 *)$ as a result of enhanced, selective intersystem crossing (Scheme 3) ${ }^{[32]}$ To rationalize the magnetic behavior of Td1* and Td2* by using a Dirac's phenomenological Hamiltonian, a three- $J$ model should, in principle, be used. There are three different, linearly arranged, magnetic sites: the two remote permanent spin carriers (SCs) and the central transient open-shell excited photosensitizer $* \mathrm{P}$ (that is, excited triplet state of An). Therefore, $J_{\mathrm{ES} 1}$ and $J_{\mathrm{ES} 2}(\mathrm{ES}=$ excited state) are supposed to describe the coupling between each of the two SCs and $* \mathrm{P}$, while $J_{\mathrm{ES}-\text { dir }}$ is defined as the direct interaction between the remote SCs (Scheme 3) through [B-*P-B] (or *[B-P-B] in Scheme 2) assembly. To simplify the problem in the case of $\mathbf{T d} \mathbf{1}^{*}$ and $\mathbf{T d} \mathbf{2}^{*}$, one can consider that $J_{\mathrm{ES} 1}$ and $J_{\mathrm{ES} 2}$ are equal for symmetry reasons. Also, contribution of the direct coupling between the SCs (that is, $J_{\mathrm{ES}-\mathrm{dir}}$ ) can be neglected, in first approximation, since its value is expected to be much smaller than $J_{\mathrm{ES}} \cdot{ }^{[32]}$ The calculation of the magnetic coupling in the excited state is then reduced to a single $J$ problem $\left(J_{\mathrm{ES}}=J_{\mathrm{ES} 1}=J_{\mathrm{ES} 2}\right)$, which is well approximated by the exchange coupling constant computed for the corresponding model dyads in the excited state (Dd 1* and Dd 2*), that is, $J_{\mathrm{ES}}$ in Scheme 3 . Such approximations have also been made for the interpretation of the experimental

Table 4. Computed isotropic hyperfine coupling constants $\left(A_{\text {iso }}\right.$, in Gauss) for selected nuclei of Td1, Td2, Dd1, and Dd 2 in their most stable spin state. For labeling scheme refer to Figure 1a.

\begin{tabular}{|c|c|c|c|c|c|}
\hline $\begin{array}{l}\text { Nuclei } \\
\text { (IN-based) }\end{array}$ & $\begin{array}{l}\text { Dd 1 } \\
(S=1 / 2)\end{array}$ & $\begin{array}{l}\text { Td1 } \\
\left(\mathrm{BS} ; m_{S}=0\right)^{[\mathrm{a}]}\end{array}$ & $\begin{array}{l}\text { Nuclei } \\
\text { (OV-based) }\end{array}$ & $\begin{array}{l}\text { Dd } 2 \\
(S=1 / 2)\end{array}$ & $\begin{array}{l}\text { Td2 } \\
\left(\mathrm{BS} ; m_{S}=0\right)^{[\mathrm{b}]}\end{array}$ \\
\hline N1 & 9.2 & $-4.6 / 4.6$ & $\mathrm{C} 1^{\mathrm{OV}}$ & -15.4 & $-7.7 / 7.7$ \\
\hline $\mathrm{C} 2^{\mathrm{IN}}$ & -14.2 & 7.1/-7.1 & $\mathrm{N} 2$ & 9.1 & $4.6 /-4.6$ \\
\hline N3 & 7.0 & $-3.5 / 3.5$ & N3 & 5.5 & $2.7 /-2.7$ \\
\hline $\mathrm{C} 4^{\mathrm{IN}}$ & -3.6 & $1.8 /-1.8$ & $\mathrm{C} 4^{\mathrm{OV}}$ & -6.2 & $-3.1 / 3.1$ \\
\hline $\mathrm{C} 5^{\mathrm{IN}}$ & -6.2 & $3.1 /-3.1$ & $\mathrm{C}(\mathrm{R})$ & -3.5 & $-1.8 / 1.8$ \\
\hline $\mathrm{O}$ & -22.2 & $-11.1 / 11.1$ & $\mathrm{O}$ & 0.7 & $0.3 /-0.3$ \\
\hline $\mathrm{C} 4^{\mathrm{Ph}}$ & 3.0 & $-1.5 / 1.5$ & $\mathrm{C} 4^{\mathrm{Ph}}$ & 3.3 & $1.7 /-1.7$ \\
\hline $\mathrm{C} 1^{\mathrm{Ph}}$ & -2.1 & 1.1/-1.1 & $\mathrm{C} 1^{\mathrm{Ph}}$ & -2.7 & $-1.4 / 1.4$ \\
\hline $\mathrm{C} 9^{\mathrm{An}}$ & 0.5 & 0.9 & $\mathrm{C} 9^{\mathrm{An}}$ & 0.7 & 0.4 \\
\hline $\mathrm{C} 10^{\mathrm{An}}$ & -0.1 & -0.9 & $\mathrm{C} 10^{\mathrm{An}}$ & -0.1 & -0.4 \\
\hline $\mathrm{N} 1\left(^{\operatorname{exptl}}\right)^{[\mathrm{c}]}$ & 9.2 & & N2 (exptl) & $12.0^{[\mathrm{d}]} / 6.49^{[\mathrm{e}]}$ & \\
\hline $\mathrm{N} 3(\operatorname{exptl})^{[\mathrm{c}]}$ & 4.2 & & N3 (exptl) & $6.2^{[\mathrm{d}]} / 5.13^{[\mathrm{e}]}$ & \\
\hline
\end{tabular}

[a] $m_{S}=0$; broken-symmetry state, $\langle S\rangle$ in Equation (3). The two values correspond to the nuclei of each of the phenylimino nitroxyl moieties. [b] $m_{S}=0$; broken-symmetry state, $\langle S\rangle$ in Equation (3). The two values correspond to the nuclei of each of the phenylverdazyl moieties. [c] From reference [32h]. [d] From reference [32f] for pyrene OV radical. [e] ENDOR data of 1,5-dimethyl-3-phenyl-6-oxoverdazyl from reference [60c]. 
data ${ }^{[32]}$ To summarize, the magnetic behavior in the excited state is therefore completely defined by a single $J$ value that has been computed by applying the BS approach to the dyad model species (Dd1* and Dd2*). This permitted to significantly reduce the computational effort and to overcome the technical problems encountered in the calculation of excited BS states in case of Td1* and of Td2*.

The $J_{\mathrm{ES}}$ values computed for Dd1* and Dd2* (Table 3) are indicative for a strong ferromagnetic coupling between the $\mathrm{SC}$ and the $* \mathrm{P}$ unit. The $J_{\mathrm{ES}}$ values are of opposite sign and two orders of magnitude larger than the values computed for the direct coupling of the SCs in the ground state (i.e., $J_{\mathrm{GS}}$ ) within corresponding triad models. These results are fully consistent with the experimental data, the excited state being ferromagnetic. ${ }^{[32 b-d, g-j]}$

The strong coupling computed is not surprising, since the two radical units ( $\mathrm{P}^{*}$ and $\mathrm{SC}$ ) strongly interact through the $\pi$ system supported by bridging phenyls. As observed in the case of the ground state, a more detailed picture of magnetic coupling phenomena can be derived from the analysis of the computed spin-density maps. In Figure 4, spin-density distributions calculated for Dd 1* and Dd $2 *$ are reported.

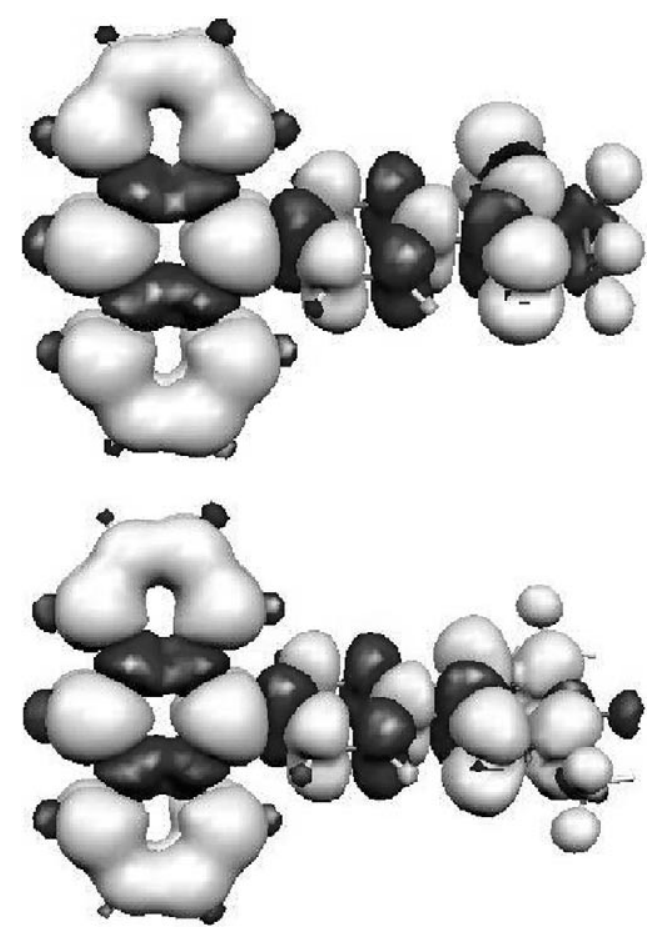

Figure 4. Spin-density patterns computed for Dd $1^{*}$ (top) and Dd 2* (bottom). $S=3 / 2$; contour value $5 \times 10^{-4}$ a.u.

First of all, significant spin density quite evenly delocalized over the anthracene backbone is observed, which corresponds to the formation of a triplet locally excited state, LES $\left({ }^{3} \mathrm{An}\right)$, as resulting from light-absorption of P. On the other hand, a spin polarization pattern is still found on both connecting extensions (the bridging phenyl elements, B) and part of the terminal SCs, in accordance with experimental findings. ${ }^{[32]}$ As compared to the ground state, the critical matching domain for the spin-density patterns of influential paramagnetic ${ }^{3} \mathrm{An}$ radical and peripheral SC has shifted from the anthracene core to the phenyl connectors. Clearly, the spin polarization phenomenon found to spread over the bridging phenyl essentially originates from the stable radical, overcoming the possible spin-delocalization effect dominant on the An entity. This prevalence of the spin polarization complies with the stronger $\pi$ conjugation of phenyl connector (B) with dangling radical $\left(\theta_{2}<3^{\circ}\right)$ than with anthracene $\left(\theta_{1} \approx 73^{\circ}\right)$, and despite excited state noticeable planarization $\left(* \theta_{1} \approx 57.5^{\circ}\right)$. As shown in Figure 4 , the energetically favored in-phase matching of the SD patterns is achieved only in the case of overall intramolecular ferromagnetic interaction corresponding to the $S=3 / 2$ state for Dd1* and Dd $2^{*}$, and according to conventional $\pi$-topological rules. ${ }^{[68]}$ Hence, a ferromagnetic interaction between the three SCs (including ${ }^{3} \mathrm{An}$ ) within Td1* and Td2*, leading to a $S=2$ excited state, can also be derived. The lowest excited-state (thexi state) of both photoexcited dyads (Ddn*) and triads (Tdn*) is computed to be ferromagnetic, according to experimental issues. Interestingly, on the same basis that is, inphase matching of spin polarization patterns over the phenyl connectors, one can also infer that, conversely to the ground state, a ferromagnetic interaction between the triplet LES of the anthracene core and the SCs is systematically favored regardless of the topological connectivity of the two [B-SC] branches with respect to $\mathrm{P}$, as exemplified by $\mathbf{T d} 3^{*}$ and Td4* (see below). These different behaviors are essentially due to the delocalized nature (with respect to inner $\mathrm{SD})$ of the central nexus LES.

Calculation of $\boldsymbol{g}$ tensors: To complete the characterization of existing Dd1,2 and Td1,2 systems, and to further establish present theoretical approach, $g$ tensors were also calculated according to the procedure described in the Computational Methods section. In the cases of Td1 and Td2, we have calculated the $g$ tensor of these systems in their ferromagnetic state $(S=1)$, although one should bear in mind that this state is only slightly higher in energy than the antiferromagnetic one and, above all, that spin multiplicity virtually has no noticeable effect over molecular geometry (see above). The results obtained are collected in Table 5 along with available experimental data.

Firstly, a general good agreement between computed and experimental data is noted. The deviation of $\Delta g_{\text {iso }}$ (that is, $g_{\text {iso }}$ vs. $\left.g_{\mathrm{e}}\right)$ with respect to $\Delta g_{\text {iso }}$ derived from experiment ranges from about $140 \mathrm{ppm}$ (case of Td1*) to roughly 440 ppm (case of An*). Such an agreement is in line with other recent work $^{[69,70]}$ demonstrating that the use of a medium-size basis set (even smaller than the Pople mix $_{\text {adopt- }}$ ed here), hybrid (or even GGA) functionals, and Koseki effective charges allow the accurate reproduction of $g$ tensors in molecular systems containing light atoms, such as the organic radicals here considered.

Secondly, $g_{\text {iso }}$ values computed for the triplet states of anthracene and diphenylanthracene are found to lie closer to 
Table 5. Computed and experimental $g$ shifts with respect to $g_{\mathrm{e}}, \Delta g$ [in ppm], in the ground and excited states.

\begin{tabular}{|c|c|c|c|c|}
\hline & \multicolumn{2}{|c|}{ An* $(S=1)$} & \multicolumn{2}{|c|}{ ph-An-ph* $(S=1)$} \\
\hline$\Delta g_{\mathrm{xx}}$ & \multicolumn{2}{|r|}{-255} & \multicolumn{2}{|r|}{239} \\
\hline$\Delta g_{\mathrm{yy}}$ & \multicolumn{2}{|r|}{466} & \multicolumn{2}{|r|}{393} \\
\hline$\Delta g_{\text {zz }}$ & \multicolumn{2}{|r|}{535} & \multicolumn{2}{|r|}{554} \\
\hline$\Delta g_{\text {iso }}$ & \multicolumn{2}{|r|}{259} & \multirow{2}{*}{\multicolumn{2}{|c|}{236}} \\
\hline \multirow[t]{2}{*}{$\Delta g_{\text {iso }}\left(\operatorname{exptl}{ }^{[\mathrm{a}]}\right)$} & \multicolumn{2}{|r|}{700} & & \\
\hline & Td1 $S=1$ & Td2 $S=1$ & Td1 $* S=2$ & Td2 $* S=2$ \\
\hline$\Delta g_{\mathrm{xx}}$ & 622 & 0 & 1379 & 452 \\
\hline$\Delta g_{\text {yy }}$ & 4025 & 1742 & 2057 & 601 \\
\hline$\Delta g_{\text {zz }}$ & 7405 & 2373 & 2971 & 1423 \\
\hline$\Delta g_{\text {iso }}$ & 4018 & 1372 & 2136 & 825 \\
\hline \multirow[t]{2}{*}{$\Delta g_{\text {iso }}\left(\operatorname{exptl}{ }^{[b]}\right)$} & & & 2000 & 1200 \\
\hline & Dd $1 S=1 / 2$ & Dd $2 S=1 / 2$ & Dd 1* $S=3 / 2$ & Dd2 $* S=3 / 2$ \\
\hline$\Delta g_{\mathrm{xx}}$ & -165 & -285 & 155 & 71 \\
\hline$\Delta g_{\text {yy }}$ & 4169 & 2271 & 1688 & 858 \\
\hline$\Delta g_{\text {zz }}$ & 8090 & 2387 & 2735 & 1102 \\
\hline$\Delta g_{\text {iso }}$ & 4032 & 1458 & 1526 & 677 \\
\hline$\Delta g_{\text {iso }}(\operatorname{exptl})$ & $3700^{[\mathrm{c}]} / 4700^{[\mathrm{a}]}$ & 1800 & $2000^{[b]}$ & $1200^{[\mathrm{d}]} / 1700^{[\mathrm{b}]}$ \\
\hline
\end{tabular}

[a] From reference [32j]. [b] From reference [32c]. [c] From reference $[32 \mathrm{~h}]$. [d] From reference $[32 \mathrm{~g}]$.

the $g$ value of the free electron (that is, smaller $\Delta g_{\text {iso }}$ ) than all other SC-derivatized systems. This finding can be qualitatively rationalized given that, in the cases of An and [phAn-ph] references, spin density is essentially localized on carbon atoms, which are lighter than the nitrogen or oxygen atoms of spin carriers (moreover supporting almost completely ASP of the molecules when present). As a consequence, spin-orbit coupling is expected to be greater for dyads and triads, resulting in larger $\Delta g_{\text {iso }}$ shifts with respect to $g_{\mathrm{e}}$. These issues are also fully consistent with experimental findings. ${ }^{[32 \mathrm{~h}-\mathrm{j}]}$

When comparing the $g_{\text {iso }}$ values computed for Td1 and Td2 to those of the corresponding monoradical species (Dd1 and Dd2), a negligible deviation is found, further substantiating the weakness of the coupling between the SCs in the ground state, which was already inferred from structural and magnetic coupling analyses. In this respect, comparison between the $g$ tensors computed for the ground and the excited states of similar systems is even more instructive. A significant reduction of the $g_{\text {iso }}$ is systematically obtained when going from the ground state to the excited state, in qualitative and quantitative consistency with the experimental data available for Dd1 and Dd2. In the case of Dd1, for instance, a reduction in $g_{\text {iso }}$ value of approximately $2600 \mathrm{ppm}$ is calculated upon excitation, in good agreement with the experimental estimate of $1700 / 2700 \mathrm{ppm} \cdot{ }^{[32 \mathrm{~h}, \mathrm{j}]}$ The decrease in $g_{\text {iso }}$ for the excited state of mono- and diradicals can be rationalized by assuming that each of the subunits involved, including the radicals (that is, the (B)-SCs, likened to Ddn) and the photoexcited anthracene core $\left(\mathrm{P}^{*}\right)$, do retain its electronic (and above all, magnetic) identity when coupling in the excited state. This assumption is confirmed by the analysis of the overall ASP of each of the P, B, and SC fragments. On the one hand, in the excited state, two electrons are fully localized on the ${ }^{3} \mathrm{An}$ unit, and one elec- tron is localized on each of the SCs. On the other hand, the phenyl B subunits are only spin-polarized by SCs and ${ }^{3} \mathrm{An}$ and thus carry virtually no net overall spin density. As a consequence, the overall $g_{\text {iso }}$ can be computed as the weighted sum of $g_{\text {iso }}$ values of individual subunits. In the case of dyads, $g_{\text {iso }}$ is therefore expressed as Equation (8) and, for triads (Tdn) as Equation (9), with $n$ being 1 or 2 (that is, Dd1/Td1 or Dd2/Td2) depending on whether IN or OV derivatives are considered.

$g_{\text {iso }}\left(\mathbf{D d} \boldsymbol{n}^{*} ; S=3 / 2\right)=\frac{g_{\text {iso }}(\mathbf{D d} \boldsymbol{n} ; S=1 / 2)+2 g_{\text {iso }}\left(\mathrm{P}^{*} ; S=1\right)}{3}$

$g_{\text {iso }}\left(\boldsymbol{T d} \boldsymbol{n}^{*} ; S=2\right)=\frac{g_{\text {iso }}(\mathbf{D d} \boldsymbol{n} ; S=1 / 2)+2 g_{\text {iso }}\left(\mathrm{P}^{*} ; S=1\right)}{2}$

Substituting computed values of Table 5 in these expressions allows us to demonstrate their fulfillment in the cases of both mono- and diradicals. Furthermore, these expressions also account for the variation of $g_{\text {iso }}$ (observed and computed) on going from the ground to the excited state, which is smaller for the verdazyl derivative (Dd2) than for the iminonitroxide monoradical (Dd1). Actually, this difference in $g_{\text {iso }}\left(\Delta g_{\text {iso(GS-ES })}\right)$ can be written as Equation (10).

$$
\begin{aligned}
\Delta g_{\text {iso }(\mathrm{GS}-\mathrm{ES})} & =g_{\text {iso }}(\mathbf{D d} \boldsymbol{n} ; S=1 / 2)-g_{\text {iso }}\left(\mathbf{D d} \boldsymbol{n}^{*} ; S=3 / 2\right) \\
& =\frac{2\left(g_{\text {iso }}(\mathbf{D d} \boldsymbol{n} ; S=1 / 2)-g_{\text {iso }}\left(\mathrm{P}^{*} ; S=1\right)\right)}{3}
\end{aligned}
$$

For the IN-based monoradical (dyad Dd1), the rather large difference $\left(\Delta g_{\text {iso(GS-ES })}\right)$ found results from a $g_{\text {iso }}$ value significantly larger (by ca. $3800 \mathrm{ppm}$ in absolute value) than that estimated for anthracene or phenylanthracene. The latter discrepancy in $g_{\text {iso }}$ stems from the larger spin-orbit coupling due to the presence of relevant spin density located on the oxygen atoms of the SC. On the other hand, given that the $g_{\text {iso }}$ value of the verdazyl monoradical (essentially involving nitrogen atoms) is only $1200 \mathrm{ppm}$ larger than that of $\mathrm{P}^{*}$, the $\Delta g_{\text {iso(GS-ES) }}$ found for $\mathrm{OV}$ derivatives is therefore significantly smaller than that calculated for the IN-based species.

Finally, we also note that the $g_{\text {iso }}$ value of photoexcited diphenylanthracene is slightly smaller than that of triplet anthracene, indicating that the SD originating from ${ }^{3} \mathrm{An}$ spreads a little over the phenyl connectors and is then shared between a larger number of nuclei. Hence, the lowering of the $\Delta g_{\text {iso }}$ also reflects the non-negligible coupling between the B connectors (ph) and the P core (An). For the excited state, to decide whether B elements should be preferably considered as part of the photosensitizer $\left(\mathrm{P}^{*}\right)$ or as extensions of the SC units is not that straightforward, especially since both the remote radicals and the ${ }^{3} \mathrm{An}$-centered triplet induce a polarization of the spin density present on the phenyls ("match/mismatch" zone). However, from viewpoint of atomic spin populations (ASP), these spin densities are rather scarce (less than 0.1 electrons per $\mathrm{B}$ ) relative to 
the ASPs of spin providers, which virtually retain their electronic (and magnetic) integrity: 1.96 electrons formally ascribed to the triplet anthracene (instead of two electrons) and 1.0 electron on average for each of the SCs. Thus, beyond considering intensity of intercomponent magnetic interaction with respect to preservation of the magnetic integrity of individual spin providers (regardless of their persistent or transient natures), the picture we get fits a localized description at the magnetic level, including in the excited state. Clearly, this is of great help for the computation and rationalization of the magnetic properties even though only their subtle interplay determines the overall magnetic behavior of the molecule. Indications regarding the magnitude of the coupling between the subunits within various molecular assemblies can be derived from the comparison of energy gaps between the lowest thexi state and the relaxed ground state $\left(\mathrm{S}_{0}\right)$ computed for the anthracene and diphenylanthracene either isolated or embedded in mono- and diradical systems (Table 6). On the one hand, the $S_{0} \rightarrow T_{1}$

Table 6. Total energy differences $(\Delta E$, in $\mathrm{eV})$ computed for anthracene (An) and 9,10-diphenylanthracene (ph-An-ph) (singlet $\left(\mathrm{S}_{0}, S=0\right)$ to triplet $\left(\mathrm{T}_{1}, S=1\right)$ ), Dd 1 and $\mathbf{D d} 2$ (doublet $\left(\mathrm{D}_{0}, S=1 / 2\right)$ to quartet $\left(\mathrm{Qr}_{1}, S=\right.$ $3 / 2)$ ), and Td1 and Td2 (triplet $\left(\mathrm{T}_{1}, S=1\right)$ to quintet $\left(\mathrm{Qn}_{1}, S=2\right)$ ).

\begin{tabular}{lllllll}
\hline & $\mathrm{An}^{[\mathrm{a}]}$ & $\mathrm{ph}^{\mathrm{Ann}-\mathrm{ph}^{[\mathrm{a}]}}$ & Dd1 1 $^{[\mathrm{a}]}$ & Dd2 $^{[\mathrm{a}]}$ & Td1 $^{[\mathrm{a}]}$ & Td2 $^{[\mathrm{a}]}$ \\
\hline $\mathrm{S}_{0}-\mathrm{T}_{1}$ & $1.81^{[\mathrm{b}]} / 2.14^{[\mathrm{c}]}$ & $1.71^{[\mathrm{b}]} / 2.01^{[\mathrm{c}]}$ & & & & \\
$\mathrm{D}_{0}-\mathrm{Qr}_{1}$ & & & $1.73^{[\mathrm{b}]}$ & $1.73^{[\mathrm{b}]}$ & & \\
$\mathrm{T}_{1}-\mathrm{Qn}_{1}$ & & & & & $1.68^{[\mathrm{b}]}$ & $1.63^{[\mathrm{b}]}$
\end{tabular}

[a] Structure optimized at LANL2 level and energy evaluate at LANL2 level. [b] Energy gap between relaxed excited and ground states. [c] Vertical transition energy.

energy gap is found to significantly decrease by about $5.5 \%$ (that is, $0.10 \mathrm{eV}$ ) on going from anthracene to diphenylanthracene deprived of radical moieties, once again indicating that the phenyl units are indeed involved in the photoexcitation process. On the other hand, regarding radical species, even though $\mathrm{S}_{0} \rightarrow \mathrm{Qr}_{1}$ energy gap calculated for monoradical is roughly the same (within $0.02 \mathrm{eV}$ ) as that obtained for isolated diphenylanthracene $\left(\mathrm{S}_{0} \rightarrow \mathrm{T}_{1}\right)$; the $\mathrm{S}_{0} \rightarrow \mathrm{Qn}_{1}$ energy gap computed for the triad diradicals is found to be smaller than for the dyads (down to $1.63 \mathrm{eV}$ for Td2* vs. $1.73 \mathrm{eV}$ for Dd 2*).

To summarize, the present theoretical approach is shown to accurately reproduce experimental ground-state observables including structural features, exchange coupling constants $J / k_{\mathrm{B}}$ and $g$ tensors reported so far for investigated polyads and related reference compounds. Our computational approach also accounts for excited-state behaviors at either level of structural (such as planarization) or magnetic properties (switching from ground-state antiferromagnetic coupling of remote SCs to a ferromagnetic one in the excited state). Finally, and most importantly, excited-state intramolecular exchange couplings could be quantitatively estimated and were found in the order of magnitude expected from experimental issues. ${ }^{[32 \mathrm{~h}, \mathrm{j}]}$ In other words, the reliability of the computational method used here has been fully established and quantitative assessment of key excited-state magnetic parameters (e.g., $J$ ) now paves the way for undertaking further examination of photomagnetic systems in view to rationally improve their performances.

\section{Functional description of photoactive $\pi$-conjugated organic molecules-towards photomagnetic molecular devices} (PMMDs): Accurate account of photomagnetic properties of diradical species was made possible by proper choice of reference functional models for both of their ground and excited state behaviors, namely diradical molecules (triads) and monoradical species (dyads), respectively. It is worth noting that the use of HDVV Hamiltonian is appropriate if these models fit a localized description with respect to their magnetic features. ${ }^{[71]}$ Interestingly, such a localized description is reminiscent of a key requirement that so-called photochemical molecular devices (PMDs) have to fit (with respect to their electronic features, in this case) into the framework of specifically developed conceptual corpus of supramolecular photochemistry. ${ }^{[14]}$ It was therefore of paramount importance to check to what extent we are dealing with multicomponent assemblies in which subunits retain their individual properties including in the excited state.

Close structure-property relationships are assuredly one of the salient features of presently studied "potentially" fully $\pi$-conjugated organic systems, so that it is worthwhile to take advantage of both structural and electronic/magnetic analytical grids to derive their functional schemes.

From a structural viewpoint, molecules are made up of three types of subunits: anthracene core (An), intervening phenyl rings (ph), and terminal dangling radicals (IN and $\mathrm{OV}$ ). There are two types of interannular direct connections represented by dihedral angles $\theta_{1}$ and $\theta_{2}$ between An, ph, and IN/OV rings, respectively (Figure 1 ). The whole assembly is potentially fully conjugated. However, from structure optimization of both ground and excited states, it appears that there exists an intramolecular steric congestion essentially located about the An-ph linkage as revealed by $\theta_{1}$ value, which is larger than $56^{\circ}$ in all cases (geometrical decoupling), also after the occurrence of the excited-state planarization process $\left(* \theta_{1}\right)$. Conversely, $\theta_{2}$ is systematically smaller than $3^{\circ}$. From an electronic viewpoint, ph is strongly conjugated with the IN/OV moiety and is therefore worth being considered as the natural extension of the dangling radical rather than the extension of the loosely conjugated An core (Scheme 2). ${ }^{[32,33 a]}$

From a functional viewpoint, stable radicals are the permanent spin carriers (SCs) in both the ground and the excited states. In the ground state, An is the photosensitizer $(\mathrm{P})$. When promoted in its excited triplet state $\left({ }^{3} \mathrm{An}\right), \mathrm{P}$ is then paramagnetic and hence works as a transient spin carrier $(* \mathrm{P}=* \mathrm{SC})$. Thus, functional schemes are not the same in the ground state and in the excited state. In the former state, the two remote SCs of diradical species interact through the closed-shell assembly made up of [ph-An-ph], which acts as the spin coupler. In the latter state, the most 
prominent interaction is by far the one mediated by intervening ph as the spin coupler, involving *SC and each of the remote SCs. It is worth noting that owing to its central location, the effective function of a nexus state is given to $* \mathrm{P}$ making possible an effective correlation (alignment) of the peripheral spins borne by SC remote radicals (Schemes 1 and 3). ${ }^{[72]}$ Whether or not phenyl bridges (B) should be considered as full functional elements like others (assign role of connectors) is not a determining concern at the present stage of the study, even though improvement of photomagnetic effect may go through optimization of the $\mathrm{B}$ element. ${ }^{[28]}$

Brought together, these issues allow the revision of the functional models for ground and excited state behaviors; hence $\left[\mathrm{SC}-\left(\theta_{2}\right)-\mathrm{B}\right]-\left(\theta_{1}\right)-[\mathrm{P}]-\left(\theta_{1}\right)-\left[\mathrm{B}-\left(\theta_{2}\right)-\mathrm{SC}\right]$ three-component assemblies (triad species, Td) and $[* \mathrm{P} / * \mathrm{SC}]-\left(* \theta_{1}\right)-\left[\mathrm{B}-\left(\theta_{2}\right)\right.$ SC] excited two-component assemblies (dyad* species, Dd*) are proposed (and used in the present work), in replacement of hitherto referred [SC]- $\left(\theta_{2}\right)-\left[\mathrm{B}-\left(\theta_{1}\right)-\mathrm{P}-\left(\theta_{1}\right)-\mathrm{B}\right]-\left(\theta_{2}\right)-[\mathrm{SC}]$ triad (Scheme 2) and *[P- $\left.\left(\theta_{1}\right)-\mathrm{B}\right]-\left(\theta_{2}\right)-[\mathrm{SC}]$ excited-dyad models, respectively. ${ }^{[32,33 a, 73]}$

Regarding suitability of a localized description of photomagnetic compounds, analysis of atomic spin population (ASP) distribution of both their ground and excited states confirms that magnetic sites (SCs and *SC anthracene triplet-state) virtually retain their "magnetic integrity" (that is, localization) regardless of the persistent or transient natures of the SCs and despite "somewhat misleading" spreading of the SD perturbation over the whole molecular backbones noticed on various spin-density maps. Triplet LES of anthracene is therefore worth to be considered as a magnetic "supersite" in the same capacity as other usual smaller magnetic sites. Clearly, sturdiness of local magnetic properties with respect to intersite magnetic (and electronic) coupling is greater than stability of local electronic properties upon increasing intercomponent electronic coupling.

ASP, that is, the amount of electron(s) present over a defined domain, is actually also informative for 1) the strength of the coupling interaction when concerning a local spin polarization about a critical intercomponent connection (linkage $)^{[32 \mathrm{~h}]}$ and 2) the spatial expanse of a magnetic site. Spindensity maps are essentially informative for 1) the mechanism, that is, inter-(magnetic)-site polarization versus on(magnetic)-site delocalization, and 2) the derived nature of (exchange) magnetic coupling (ferro/antiferro, depending on resulting SD interference pattern).

Magnetic sites appear to be spatially more localized than corresponding electronic counterparts. Thus, the excited triplet state of anthracene is a locally excited state (LES) from an electronic (photophysical) viewpoint, but involves its phenyl substituents to a great extent. This point, visualized by spin-density maps, is also revealed by 1) excitedstate planarization about $\left.\theta_{1}, 2\right)$ computed decrease of $g_{\text {iso }}$ upon photoexcitation of isolated diphenylanthracene, and 3) decrease of energy gap between relaxed ground state and triplet excited state on going from An to [ph-An-ph]. Nonetheless, with regard to its actual spatial extent, the corre- sponding ASP is virtually confined to the anthracene core over which SD is furthermore delocalized. Because of its respectable spatial extension (the whole An backbone) with respect to usual magnetic sites (e.g., IN), triplet LES $\left({ }^{3} \mathrm{An}\right)$ is quoted as a magnetic "supersite". Also, it has been demonstrated that the variation, upon excitation, of $g_{\text {iso }}$ values attached to dyads could be accurately computed from the weighted contributions of their parent components (namely, the SC within the dyad and $* \mathrm{P}$ ). This point is a hallmark of supermolecules, that is, of weakly coupled multicomponent systems. ${ }^{[14]}$ The status of the phenyl B subunits with respect to ASP is different from that of magnetic sites. The spin-polarization pattern of ASP present on each phenyl groupand preferably located at nuclei directly proximal to the An core-is partly responsible for quite significant magnetic coupling (ca. $+80 \mathrm{~cm}^{-1}$ ) between central paramagnetic triplet state and remote doublet state of persistent radicals. However, the overall ASP attached to these phenyls is negligible $\left(<0.1 \mathrm{e}^{-}\right)$. The same localized description also holds for the ground state of diradical species, but, in this case, it only concerns the stable paramagnetic terminal sites. The point is that magnetic coupling is sufficiently small not to endanger magnetic identity of interacting sites (SC and *SC) in the same manner as electronic interaction is adequately weak to save electronic integrity of components in PMDs assemblies. ${ }^{[14]}$ As a matter of fact, the critical threshold for magnetic interaction is dramatically higher than for electronic coupling.

The above-derived issues concerning actual identity of magnetic (super)sites, their preserved magnetic integrity within excited assemblies, and the contribution of phenyl B subunits is further confirmed by careful analysis of active molecular orbitals (MOs; see Supporting Information). In particular, the localized nature of various magnetic sites as well as their relative spatial extents can be derived from the shape of their respective singly occupied MOs (SOMOs). Thus, magnetic site of IN (within Dd1) is essentially composed of its nitroxyl (NO) and imino nitrogen atoms (Figure SI-2), while that of OV (within Dd2) is made up of its four atoms (Figure SI-3). SOMOs attached to ${ }^{3}[\mathrm{ph}-\mathrm{An}-\mathrm{ph}]$ are composed of up to ten of the 14 carbon atoms of the An backbone and virtually none of the two phenyl groups (Figure SI-4), hence, again, ${ }^{3}$ An denotes a magnetic "super"-site. Therefore, magnetic sites are actually only parts of the SC functional components.

The overall picture we get is illustrated in Scheme 4, for the favorable (i.e., energy advantaged) case of in-phase matching of spin-polarization patterns attached to various magnetic sites. The spin coupler(s) refers to part(s) of the molecular backbone supporting a (super)exchange pathway(s) between magnetic sites, that is, undergoing spin polarization. Phenyl B units are parts of the spin coupler(s) in both the ground and excited states; An is part of the spin coupler in the ground state only.

Thus, the relevance of a Heisenberg-Dirac treatment of the (photo)magnetic properties of the supermolecules ${ }^{[14]}$ currently investigated is confirmed as we are dealing with mul- 


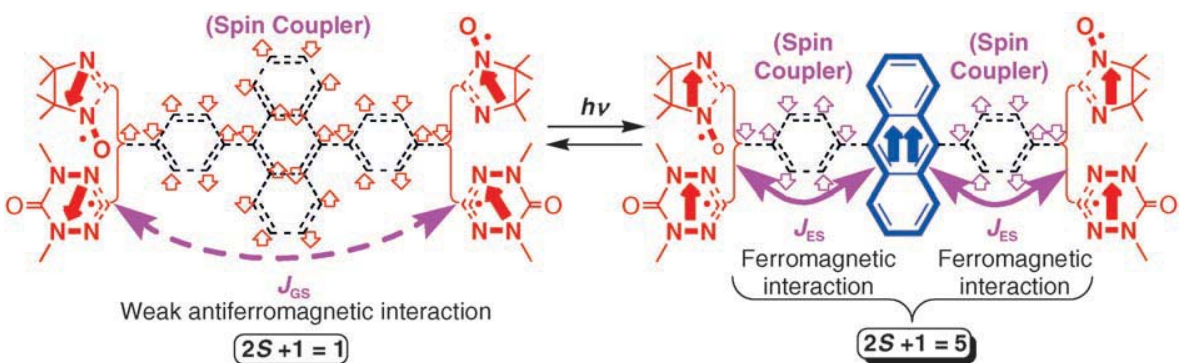

Scheme 4. The energy-advantaged in-phase matching of spin-polarization patterns: Spin coupler: (dotted); magnetic (super)sites: bold; spin-polarization patterns (empty arrows); $J_{\text {ES-dir }}$ (Scheme 3 ) is negligible.

doing so, the only parameter varied in the excited state is therefore the strength of the coupling between $* \mathrm{SC}$ (that is, *P) and the remote SCs, which depends for the most part on $\theta_{1}{ }^{[74]}$ According to refined descriptions of PMMDs provided above, no such favorable change of the key excited-state exchange coupling can be expected by alternatively varying the connection scheme of the dangling SCs about the B con-

ticomponent systems (localized description; Schemes 3 and 4).

Finally, the overall functional picture we get by applying analytical grid perfected for PMDs leads us to propose extending and transposing the latter paradigm to the herein investigated magnetic systems, so that we can now refer to photomagnetic molecular devices (PMMDs) with adjustments (on a rise) of the critical level of intercomponent (electronic-magnetic) coupling.

\begin{abstract}
Alternative topological connectivity for iminonitroxide derivatives-Td3, Td4, Dd3, and Dd4: Once the relevance of the theoretical modeling was checked against available experimental data ${ }^{[32 \mathrm{~b}-\mathrm{d}, \mathrm{g}-\mathrm{j}]}$ and the functional picture of photoresponsive supermolecules was refined, computational tools were used to investigate the contribution of the various parameters to photomagnetic behavior, intending to improve the efficiency of the PMMDs, for example, by increasing exchange coupling in the excited state. As already underscored for the potentially fully $\pi$-conjugated systems investigated, structure-property relationships are all the more prominent, since functional behavior is largely governed by intercomponent couplings and semirigid architectures allow conformational fluctuations. ${ }^{[55-57,66 a]}$ Given that SD distribution attached to the triplet locally excited state of $* \mathrm{P}$ is roughly uniform, that is, of the same sign regardless of the connection site of An core, it was therefore interesting to vary the topological connectivity (Figure 1b) with an eye to changes of the dihedral angle $\theta_{1}$ related to a steric decongestion about the canted planes of the $\mathrm{P}$ core and $\mathrm{B}$ connectors. In
\end{abstract}

nectors (essentially affecting downward optimum value of $\theta_{2}$ twist angle). ${ }^{[32 \mathrm{a}, \mathrm{c}, \mathrm{e}, \mathrm{h}, 75]}$

Ground- and excited-state magnetic behavior of two bisIN derivatives (Td3 and Td4, Figure 1b) with alternative connection schemes, which have not yet been studied experimentally, were analyzed theoretically. Structural data computed for these diradicals as well as for affiliated monoradicals (Dd3 and Dd4, Figure 1b) are reported in Table 7 and in the Supporting Information (Table SI-III). In contrast to systems Td1 and Td2, remote SCs within Td3 and Td4 are no longer symmetry related.

From inspection of Table 7 it appears that structural parameters of the iminonitroxide moieties are almost the same as those computed for the phenyl-substituted IN radical, regardless of whether the latter is isolated or embedded in Td1 or Dd1. Furthermore, for biradical systems Td3 and Td4, there is a negligible effect of the overall spin multiplicity on the internal structural parameters of the SCs, as already underscored for Td1 and Td2. Thus, SC subunits retain their identity at the structural level in both cases of the radical and biradical systems. Nonetheless, there are significant structural differences for the new topologies, in particular for Td4 and Dd4. In the ground state, smaller $\theta_{1}$ values are computed for the phenyl group connected to the anthracene unit at position $\mathrm{C}^{\mathrm{An}}$ (ca. $52^{\circ}$ for Td3 and Dd3) or $\mathrm{C}^{\mathrm{An}}$ (ca. $33^{\circ}$ for Td4 and Dd4) as compared to $\theta_{1}$ value for phenyl group connected at position $\mathrm{C} 10^{\mathrm{An}}$, which keeps the same conformation as that computed within affiliated Td 1 and Dd $1\left(\theta_{1} \approx 72^{\circ}\right)$. Clearly, in the ground state, this angular disparity is not related to electronic effects, since the

Table 7. Selected structural parameters computed for iminonitroxide derivatives Td3/Td4 and Dd3/Dd4 in the ground state (Td3, Dd3, Td4, Dd4) and in the excited state $\left(\mathbf{T d 3}^{*}, \mathbf{D d}^{*}, \mathbf{T d} 4^{*}, \mathbf{D d} 4^{*}\right)$ as a function of the overall spin multiplicity. Distances in $\AA$, angles in degrees. For labeling scheme refer to Figure $1 b$.

\begin{tabular}{|c|c|c|c|c|c|c|c|c|c|c|}
\hline & $\begin{array}{l}\mathbf{T d ~ 3}^{[\mathrm{a}]} \\
\left(\mathrm{BS} ; m_{S}=0\right)\end{array}$ & $\begin{array}{l}\mathbf{T d 3}^{[\mathrm{a}]} \\
S=1\end{array}$ & $\begin{array}{l}\mathbf{T d 3}^{*[\mathrm{a}]} \\
S=2\end{array}$ & $\begin{array}{l}\text { Dd3 } \\
S=1 / 2\end{array}$ & $\begin{array}{l}\text { Dd 3* } \\
S=3 / 2\end{array}$ & $\begin{array}{l}\mathbf{T d} 4^{[\mathrm{b}]} \\
\left(\mathrm{BS} ; \mathrm{m}_{S}=0\right)\end{array}$ & $\begin{array}{l}\mathbf{T d}^{[\mathrm{b}]} \\
S=1\end{array}$ & $\begin{array}{l}\text { Td4*[b] } \\
S=2\end{array}$ & $\begin{array}{l}\text { Dd4 } \\
S=1 / 2\end{array}$ & $\begin{array}{l}\text { Dd 4* } \\
S=3 / 2\end{array}$ \\
\hline$d \mathrm{C} 9^{\mathrm{An}}-\mathrm{C} 1^{\mathrm{Ph}}$ & $1.493 / 1.487$ & $1.493 / 1.487$ & $1.477 / 1.479$ & 1.487 & 1.478 & $1.471 / 1.469$ & $1.471 / 1.469$ & $1.478 / 1.475$ & 1.481 & $\overline{1.473}$ \\
\hline$d \mathrm{C} 4^{\mathrm{Ph}}-\mathrm{C} 2^{\mathrm{IN}}$ & $1.471 / 1.470$ & $1.471 / 1.470$ & $1.467 / 1.468$ & 1.470 & 1.468 & $1.471 / 1.469$ & $1.471 / 1.469$ & $1.468 / 1.467$ & 1.469 & 1.467 \\
\hline$d \mathrm{C} 2^{\mathrm{IN}}-\mathrm{N} 1$ & $1.419 / 1.419$ & $1.419 / 1.419$ & $1.418 / 1.419$ & 1.419 & 1.419 & $1.419 / 1.419$ & $1.419 / 1.419$ & $1.418 / 1.419$ & 1.419 & 1.419 \\
\hline$d \mathrm{C} 2^{\mathrm{IN}}-\mathrm{N} 3$ & $1.311 / 1.311$ & $1.311 / 1.311$ & $1.313 / 1.313$ & 1.311 & 1.313 & $1.311 / 1.311$ & $1.311 / 1.311$ & $1.313 / 1.313$ & 1.311 & 1.313 \\
\hline$\theta_{1}$ & $-72.2 /-51.8$ & $-72.2 /-51.8$ & $-56.3 /-44.6$ & -51.8 & -44.4 & $-72.2 / 33.4$ & $-72.2 / 33.4$ & $-57.3 / 28.6$ & 33.3 & 27.8 \\
\hline$\theta_{2}$ & $0.7 / 1.3$ & $0.7 / 1.3$ & $1.6 /-1.0$ & 1.9 & 1.7 & $1.1 /-0.5$ & $1.1 /-0.5$ & $1.1 /-1.2$ & -1.2 & -0.8 \\
\hline
\end{tabular}

[a] The first value corresponds to the iminonitroxide linked at position $\mathrm{C} 10^{\mathrm{An}}$; the second to that at position $\mathrm{C} 1^{\mathrm{An}}$. [b] The first value corresponds to the iminonitroxide linked at position $\mathrm{C} 10^{\mathrm{An}}$, the second to that at position $\mathrm{C} 2^{\mathrm{An}}$. 
overall spin-density population (ASP) on the anthracene unit is unchanged relative to that found for Td1 and Dd1. Actually, the structure flattening of Td3 and Td4 derivatives can be explained in terms of differential steric hindrance. Steric repulsion between hydrogen atoms of the phenyl spacers and peri-hydrogen $(p-\mathrm{H})$ atom(s) of adjacent ring(s) of the anthracene platform is gradually increasing when going from $\mathrm{C}^{\mathrm{An}}$ (no $p-\mathrm{H}$ atom; Dd4) to $\mathrm{C}^{\mathrm{An}}$ (one $p-\mathrm{H}$ atom; Dd 3) and $\mathrm{C} 10^{\mathrm{An}}$ (two $p-\mathrm{H}$ atoms; Dd1; Figure 1), in qualitative agreement with the computed $\theta_{1}$ values.

Upon photoexcitation, the main sizable structural effect is the planarization ${ }^{[62]}$ about $\theta_{1}$, which is computed to be of similar amplitude for various dyads Dd1, Dd3, and Dd4, that is, a reduction of $\theta_{1}$ of approximately $15-20 \%$ subsequent to spin-density redistribution.

In summary, from a structural point of view, derivatives Td3 and Td4 are very similar to their parent compound Td1, although smaller steric constraints make a larger coupling possible between the SCs through intervening B connectors and $\mathrm{P}$ units in both the ground and the excited states.

The magnetic behavior of the $\mathbf{T d} \mathbf{3}$ and $\mathbf{T d} \mathbf{4}$ derivatives, in the ground and in the excited states, can be rationalized along the same lines followed for Td1 and Td2. The $J_{\mathrm{GS}}$ values obtained for $\mathbf{T d} 3$ and $\mathbf{T d} 4$, are reported in Table 8.

Table 8. Computed intramolecular exchange coupling constants in the ground state for Td4 and Td3 $\left(J_{\mathrm{GS}} / k_{B}\right.$, in $\left.\mathrm{K}\right)$ and in the excited state for Dd 4* and Dd 3* $\left(J_{\mathrm{ES}} / k_{B}\right.$, in K). Negative $J$ values imply antiferromagnetic coupling.

\begin{tabular}{lllll}
\hline & Td3 & Td4 & Dd3* & Dd4* \\
\hline$J_{\mathrm{GS}} / k_{B}$ & -0.1 & +0.5 & & \\
$J_{\mathrm{ES}} k_{B}$ & & & +83 & +100 \\
\hline
\end{tabular}

As expected, the two SCs are loosely coupled in the ground state through the closed-shell diphenylanthracene network, which provides a poor magnetic superexchange pathway. Although the broken symmetry approach is not precise enough to allow a quantitative discussion of these small $J$ values (as already mentioned for Td1 and Td2), the sign of $J$ (referring to ferro- or antiferromagnetic coupling) can safely be determined from this method. Thus, the iminonitroxide radicals in $\mathbf{T d} \mathbf{3}$ are found to be antiferromagnetically weakly coupled $\left(J_{\mathrm{GS}} / k_{\mathrm{B}}=-0.1 \mathrm{~K}\right)$, like in Td1 and conversely to Td4, in which they are found to weakly couple ferromagnetically $\left(J_{\mathrm{GS}} / k_{\mathrm{B}}=+0.5 \mathrm{~K}\right)$ even in the ground state. These issues are in agreement with the mechanistic picture we got from the spin-density map computed for Dd1. Indeed, in the case of the connection scheme of Td4, a constructive interference pattern over the anthracene core, that is, in-phase matching of the spin polarizations originating from the radicals (and mediated by the phenyl connectors), is found only when the two iminonitroxide radicals are ferromagnetically coupled (Figure 5). Antiferromagnetic layout of the dangling radicals results in an energetically disfavored phase-mismatch of the spin-density patterns in par-
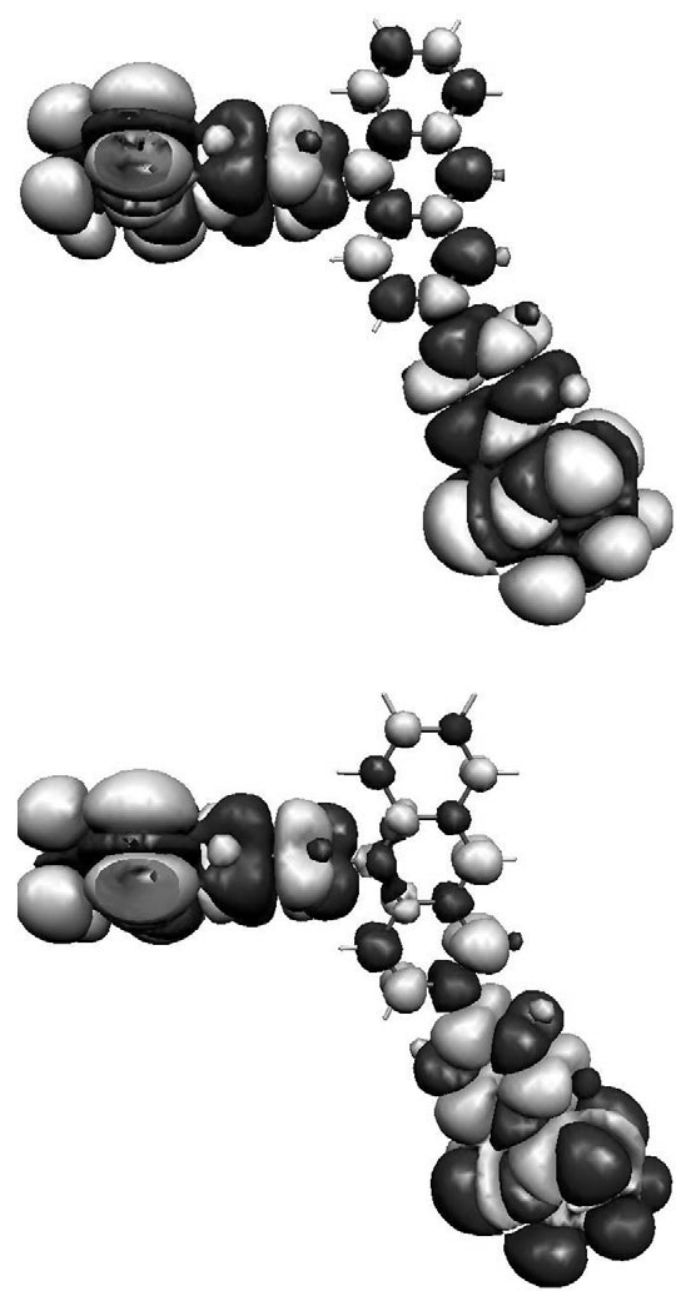

Figure 5. Spin-density patterns computed for the triplet state $(S=1$; top) and the BS state ( $m_{\mathrm{S}}=0$; bottom) for Td4 in the ground state (contour value $5 \times 10^{-5}$ a.u.).

ticular at positions $\mathrm{C} 10^{\mathrm{An}}$ and $\mathrm{C} 2{ }^{\mathrm{An}}$. This destructive interference is also revealed by overall lowering of the spin polarization found on the An domain (Figure 5).

In the excited state, the coupling between the triplet excited state of the anthracene magnetic supersite, and the two iminonitroxide magnetic sites should now be described by two different exchange coupling constants $J_{\mathrm{ES} 1}$ and $J_{\mathrm{ES} 2}$, respectively (Scheme 3). The first one accounts for the interaction with the iminonitroxyl linked at position $\mathrm{C} 10^{\mathrm{An}}$ and the second one is for the SC connected through intervening phenyl groups at positions $\mathrm{C}^{\mathrm{An}}$ (for $\mathbf{T d} 3^{*}$ ) or $\mathrm{C}^{\mathrm{An}}$ (for Td4*). By applying the same approximation as for the case of Td1, that is, by neglecting the contribution of the direct interaction between the remote SCs ( $J_{\mathrm{ES}-\mathrm{dir}}$; Scheme 3$)$, the coupling between each of the spin carrier and the triplet anthracene can be approximated by the exchange coupling constant computed, in the excited state, for the corresponding P-(B)-SC dyad systems; that is, Dd1* for $J_{\mathrm{ES} 1}$ (Table 3) and Dd 3* or Dd 4* for $J_{\mathrm{ES} 2}$ (Table 8 ). As expected on the basis of the spin-density maps computed for the quartet excited state of the model dyads, the coupling is ferromagnetic 
in all cases and consequently also for affiliated triads. However, excited-state magnetic-exchange pathways related to alternative connection schemes of Td3 and Td4 are found to be less efficient $\left(J_{\mathrm{ES} 2} / k_{\mathrm{B}}=+91.5 \mathrm{~K}\right.$ on average $)$ than that of Td1 and Td2 relying on the usual arrangement the SCs along the $\mathrm{C} 9{ }^{\mathrm{An}}-\mathrm{C} 10^{\mathrm{An}}$ axial positions $\left(J_{\mathrm{ES} 1} / k_{\mathrm{B}}=+127 \mathrm{~K}\right)$ in spite of the reduced steric hindrance about $\theta_{1}$ (strengthened $\mathrm{P}-\mathrm{B}$ coupling).

Close inspection of spin-density maps computed for the lowest thexi state of various polyads, including reference anthracene and diphenylanthracene, reveals that the spin density is not that evenly distributed over the anthracene backbone. This pattern is of similar shape in all cases, hence it is an intrinsic feature of the triplet LES of the acene core. As already noted by Teki, ${ }^{[32 \mathrm{~h}]}$ ASPs located at key connection sites of the central ring (that is, ca. $0.75 \mathrm{e}^{-}$at each of the $\mathrm{C} 9^{\mathrm{An}}$ and $\mathrm{C} 10^{\mathrm{An}}$ positions $)^{[76]}$ are much larger than ASPs found at $\mathrm{C}^{\mathrm{An}}$ (ca. $0.23 \mathrm{e}^{-}$) or $\mathrm{C}^{\mathrm{An}}$ sites (ca. $0.07 \mathrm{e}^{-}$) of the side rings, while it is recognized that magnitude of $J$ is also related to amount of spin density mobilized for exchange interactions. ${ }^{[77]}$ Brought together, these findings may explain that, in the excited state, exchange pathways relying on two different rings (even directly fused), that is, including at least one of the side rings (as is typically the case for connection schemes of Td3 and Td4), are less efficient than exchange channel of Td1 and Td2 relying on the sole central ring of anthracene.

General comments: First of all, the present work was aimed at quantitatively assessing key parameters-essentially magnetic exchange coupling constants $(J)$-allowing accurate description of photoinduced, intramolecular, spin-alignment process that occurs within purpose-designed open-shell organic molecules. Determination of excited-state $J$ values $\left(J_{\mathrm{ES}}\right)$ was achieved by the successful use of the HDVV Hamiltonian thanks to the relevance of the localized description of basic magnetic features at the molecular level. Indeed, the localized nature of the magnetic sites embedded in the $\pi$-delocalized molecular framework has been established, and found to be independent of their persistent or transient, slightly more diffuse (e.g. triplet LES-termed magnetic "supersite"), natures. Hence, by naturally drawing our inspiration from the paradigm of so-called photochemical molecular devices (PMDs), pertinent model systems for groundand excited-state behaviors were derived together with the definition of a new class of photoresponsive multicomponent assemblies, showing light-driven changes of their magnetic features, namely, photomagnetic molecular devices, PMMDs.

From a more qualitative viewpoint, we found that recourse to terminology normally used for waves through pictorial "interference of polarized SD distributions" made long-range magnetic interaction between two or more localized magnetic sites embedded in a rather large $\pi$-delocalized framework (that is, in a potentially fully conjugated system) more straightforwardly understandable. This representation allows us to take into account both magnetic sites and super- sites as well as the fading of the ASPs with the distance for long-range interactions, which is not explicitly considered when qualitatively applied (to short-range interaction) conventional $\pi$-topological rules. ${ }^{[11,68]}$ Here, the "wavy" feature of the SD actually refers to the particular pattern of SD distribution attached to a single paramagnetic (super)site and characterized by sign alternation as a result of antiferromagnetic interaction between neighboring sites of the $\pi$ system due to on-site Coulomb repulsion (polarization mechanism). Amplitude attached to these "wavy" (polarized) SD distributions spreading over the $\pi$-delocalized system, would be essentially defined at each site (atom) of the $\pi$ system as the absolute value of $(\alpha-\beta)$ net electron-spin contribution that is, the ASP, and would be reducing with the distance to the SD epicentre (that is, the magnetic site location, either mono- or oligo- to multiatomic as is the case for triplet of anthracene "supersite"). Hence, superposition of two or more SD "wavy" distributions, each attached to odd electron spins, results in a new SD pattern corresponding to actual superexchange pathway for interacting magnetic sites (Figures 3 and 5), which can either be ferro- or antiferromagnetic. Depending on the matching or the mismatching of the SD distribution patterns, pictorially referred to as constructive or destructive interferences, ASP attached to the new pattern-and defined at each position of the $\pi$ framework -is enlarged or diminished accordingly, which is directly related to the magnitude of the resulting exchange coupling. It is worth noting that destructive interference of SD patterns results in an energy disadvantaged coupling, regardless of its ferro- or antiferromagnetic nature. To summarize, this qualitative and somewhat pictorial approach allowed us to account for both the ground- and excited-state magnetic behaviors of multispin systems relying on longrange to short-range interactions, mediated by a $\pi$-delocalized framework (superexchange coupling) including "conformational defects" (e.g., interannular twist angles such as $\theta_{1}$ ).

Amongst the various possible $\mathrm{P} / \mathrm{B} / \mathrm{SC}$ connection schemes explored by $\mathrm{us}^{[78]}$ and others, ${ }^{[32 \mathrm{a}-\mathrm{e}, \mathrm{g}-\mathrm{g}]} \mathrm{B}$ functionalization at the $\mathrm{C} 9^{\mathrm{An}}$ - and $\mathrm{C} 10^{\mathrm{An}}$-positions of $\mathrm{P}$ is found to be more efficient for generating photoinduced intramolecular spin alignment, despite the detrimental effect of greater $\mathrm{P} / \mathrm{B}$ intercomponent steric hindrance (reflected by $\theta_{1}$ ) upon magnetic coupling. Actually, partial localization of $\mathrm{SD}$ at $\mathrm{C} 9^{\mathrm{An}} / \mathrm{C} 10^{\mathrm{An}}$ positions more than compensates for adverse opposite contribution of geometrical decoupling, even if lowered on occasion of transient structural relaxation process, namely, planarization. Large ASP values attached to $\mathrm{C} 9^{\mathrm{An}}$ - and $\mathrm{C} 10^{\mathrm{An}}{ }_{-}$ positions of triplet anthracene also give to these sites peculiar sensitivity, for instance revealed by preferential location for photodimerization process and Dewar valence isomerism. ${ }^{[36]}$

Most importantly, detailed analysis of ground- and excited-state magnetic behaviors of corresponding biradical species exhibiting symmetric (Td1,2) and asymmetric (Td3,4) intramolecular connection schemes shows that photosensitive anthracene is a suitable functional element to induce effective ferromagnetic alignment (correlation) of spin carriers 
lying at its periphery when promoted in its triplet excited state. These findings open up new guidelines for the design of photoresponsive multispin systems composed of more than two permanent SCs arranged about the acene core and giving rise to metastable high-spin molecules (Scheme 1a). Indeed, beyond structural symmetry of the anthracene center, the natural uniformity of the SD pattern attached to the nexus excited state (activated magnetic "supersite") is of crucial importance. However, full exploitation of the connection potential of anthracene, as a dendritic connector or a molecular hub, by multisite derivatization is anticipated to dramatically distort the structure of the acene backbone. ${ }^{[79]}$ Such a distortion is also likely to alter (but not that much ${ }^{[79]}$ the key inner delocalized pattern of SD of the nexus state and individual $\mathrm{P}-\mathrm{B}$ coupling interactions, even though the latter are expected to remain correlated. Bridging (B) elements connectors not only mediate magnetic communication between remote SCs and the central nexus core, they also act as spacers, easing multiderivatization by reducing steric congestion in the direct surrounding of $\mathrm{P}$ (Scheme 1). ${ }^{[80]}$ Therefore, B elements are bound to be a prominent part in the design of the new PMMDs. In a forthcoming paper, we will show that photomagnetic performances are indeed markedly improved by replacing a $p$-phenylene connector by an heterocyclic building block. ${ }^{[28,81]}$ Applications of such PMMDs are envisioned in the field of molecular spintronic devices. ${ }^{[82]}$ Indeed, on the one hand, photoinduced alignment of intramolecular spins can be likened to an electron-spin polarization transfer from a unique excitonic trigger to originally uncorrelated (or loosely correlated) spins of dangling radicals and, on the other hand, the resulting in-phase correlation of peripheral spins (spin alignment) can be viewed as the transposition at the intramolecular level of spin coherences produced in semiconductor-based spintronic devices. $^{[83]}$

\section{Conclusion}

Through-space interactions, whether inter- or intramolecular, are ubiquitous in molecule-based magnetic materials and are recognized to be sometimes even determining. For photomagnetic molecular devices (PMMDs), magnetic properties are essentially governed by intramolecular interactions and in particular by through-bond mediated coupling. This feature makes it possible to achieve their rational design according to an approach similar to that developed for photochemical molecular devices (PMDs). The design method is based on a reductionist analysis of the targeted function and consists of identifying different candidates for basic component-related properties that could be associated. ${ }^{[14]}$ Therefore, successful application of conceptual precepts of supramolecular photochemistry adapted to magnetic properties (photomagnetism) partly relies on the nature of bridging elements (connectors B). Indeed, beyond the architectural rigidity that they have to ensure, intervening $\mathrm{B}$ elements are in the present case supposed to mediate rather strong magnetic coupling, such as the exchange coupling as compared to the dipolar interaction. Phenylene units, regardless of their ortho/meta/para connection schemes, are thus particularly well suited as connectors.

There is no net SD transport neither is there transfer through the bridging connector of magnetic sites; that is, there is no charge transfer (CT) character. The magnitude of exchange coupling of the magnetic sites is essentially determined by the amplitude of polarization (characterized by local ASP) at the connecting sites, that is, about intercomponent (SC-B and $\mathrm{B}-* \mathrm{SC} /{ }^{3} \mathrm{P}$ ) linkages, but in no way it is determined by integrated value of SD or ASP present over the connector domain, which remains equal to zero. Polarization is worth being viewed as a perturbation that locally roughly compensates over two adjacent sites (sign alternation), solely mediating magnetic information. Whether or not intramolecular polarization at the electronic level induced by bridging elements (and giving partial CT character to the multicomponent system) is likely to enhance SD polarization pattern, thus rendering the magnetic exchange coupling more efficient, deserves to be clarified. ${ }^{[81]}$

Purely organic systems and attached magnetic supersites (such as the triplet state of An) have the great asset over inorganic cores that they show more potential as dendritic connectors (nexus) than metal ions, which are limited by their coordination number.

Finally, the PMMDs here studied can be referred to as two-branched, photoactivable "proto-dendritic connectors" (in the case of triad assemblies), and are amongst the most promising type of multifunctional system aimed at playing a role in the transposition of conventional spintronics (based on microchips) into molecular-scale devices: towards (supra)molecular spintronics.

\section{Acknowledgements}

The IDRIS (French national computer center) is gratefully acknowledged for computer time allocation within the Project 072115. I.C., P.P.L., C.A.D., and C.A. are grateful to European Egide integrated action program "Germaine de Staël" (PAI-10612NL) for financial support. P.P.L. and V.M. are also indebted to the French ministry of research for funding (ACI project no. JC4123). I.C., V.M., and C.A. are grateful to the Paris 6 University for funding (UPMC-BQR 2006). The authors thank Dr. Gaston Berthier for fruitful discussions.

[1] a) O. Sato, T. Iyoda, A. Fujishima, K. Hashimoto, Science 1996, 272, 704-705; b) M. Verdaguer, Science 1996, 272, 698-699.

[2] a) J.-F. Létard, P. Guionneau, O. Nguyen, J. S. Costa, S. Marcén, G. Chastanet, M. Marchivie, L. Goux-Capes, Chem. Eur. J. 2005, 11, 4582-4589; b) S. Bonhommeau, G. Molnar, A. Galet, A. Zwick, J.A. Real, J. J. McGarvey, A. Bousseksou, Angew. Chem. 2005, 117, 4137-4141; Angew. Chem. Int. Ed. 2005, 44, 4069-4073; c) O. Sato, J. Photochem. Photobiol. C 2004, 5, 203-223, and references therein; d) P. Gütlich, Y. Garcia, T. Woike, Coord. Chem. Rev. 2001, 219$221,839-879$.

[3] For multifunctional molecule-based magnetic materials in general, see for instance: a) J. S. Miller, Dalton Trans. 2006, 2742-2749; b) A. B. Gaspar, V. Ksenofontov, M. Seredyiuk, P. Gütlich, Coord. Chem. Rev. 2005, 249, 2661-2676; c) E. Coronado, P. Day, Chem. 
Rev. 2004, 104, 5419-5448; d) S. Decurtins, R. Pellaux, G. Antorrena, F. Palacio, Coord. Chem. Rev. 1999, 190-192, 841-854.

[4] Selected references beyond photomagnetism: a) D. B. Amabilino, J. Veciana, Top. Curr. Chem. 2006, 265, 253-302; b) M. Ruben, U. Ziener, J.-M. Lehn, V. Ksenofontov, P. Gütlich, G. B. M. Vaughan, Chem. Eur. J. 2005, 11, 94-100; c) L. Ouahab, Chem. Mater. 1997, 9, 1909-1926.

[5] Light-induced excited spin state trapping (LIESST effect): a) S. Decurtins, P. Gütlich, C. P. Köhler, H. Spiering, A. Hauser, Chem. Phys. Lett. 1984, 105, 1-4; b) A. Hauser, Chem. Phys. Lett. 1986, 124, 543-548; ligand-driven light-induced spin change (LD-LISC effect): c) C. Roux, J. Zarembowitch, B. Gallois, T. Granier, R. Claude, Inorg. Chem. 1994, 33, 2273-2279; d) M.-L. Boillot, A. Sour, P. Delhaès, C. Mingotaud, H. Soyer, Coord. Chem. Rev. 1999, 190-192, 47-59.

[6] Photomagnetic high-spin molecules: a) J. M. Herrera, V. Marvaud, M. Verdaguer, J. Marrot, M. Kalisz, C. Mathonière, Angew. Chem. 2004, 116, 5584-5587; Angew. Chem. Int. Ed. 2004, 43, 5468-5471; b) V. Marvaud, J. M. Herrera, T. Barilero, F. Tuyèras, R. Garde, A. Scuiller, C. Decroix, M. Cantuel, C. Desplanches, Monatsh. Chem. 2003, 134, 149-163.

[7] I. Ratera, D. Ruiz-Molina, J. Vidal-Gancedo, J. J. Novoa, K. Wurst, J.-F. Létard, C. Rovira, J. Veciana, Chem. Eur. J. 2004, 10, 603-616, and references therein.

[8] J. A. Crayston, J. N. Devine, J. C. Walton, Tetrahedron 2000, 56, $7829-7857$.

[9] D. A. Dougherty, Acc. Chem. Res. 1991, 24, 88-94.

[10] a) C. Kollmar, O. Kahn, Acc. Chem. Res. 1993, 26, 259-265; b) O. Kahn, Molecular Magnetism; VCH: New York, 1993.

[11] A. Rajca, Chem. Rev. 1994, 94, 871-893.

[12] a) J. S. Miller, Adv. Mater. 2002, 14, 1105-1110; b) J. S. Miller, A. J. Epstein, Angew. Chem. 1994, 106, 399-432; Angew. Chem. Int. Ed. Engl. 1994, 33, 385-415.

[13] a) D. Gatteschi, R. Sessoli, Angew. Chem. 2003, 115, 278-309; Angew. Chem. Int. Ed. 2003, 42, 268-297; b) D. Gatteschi, $A d v$. Mater. 1994, 6, 635-645; c) D. Gatteschi, Molecular Magnetic Materials; Kluwer Academic Publishers, Amsterdam, 1991.

[14] a) V. Balzani, Tetrahedron 1992, 48, 10443-10514; b) V. Balzani, F. Scandola, Supramolecular Photochemistry, Ellis Horwood, Chichester (UK), 1991, Chapter 12; c) V. Balzani, L. De Cola, L. Prodi, F. Scandola, Pure Appl. Chem. 1990, 62, 1457-1466; d) V. Balzani, L. Moggi, F. Scandola, in Supramolecular Photochemistry (Ed.: V. Balzani), Reidel, Dordrecht (The Netherlands), 1987; pp. 1-28.

[15] Special issue "Photochromism: Memories and Switches", M. Irie, (Guest Editor) Chem. Rev. 2000, 100, 1683-1890, and references therein.

[16] a) D. Gust, T. A. Moore, A. L. Moore, Chem. Commun. 2006, 11, 1169-1178, and references therein; b) D. Gust, T. A. Moore, A. L. Moore, Acc. Chem. Res. 2001, 34, 40-48.

[17] a) M. R. Wasielewski, J. Org. Chem. 2006, 71, 5051-5066, and references therein; b) M. R. Wasielewski, Chem. Rev. 1992, 92, 435-461.

[18] J. M. Cole, Chem. Soc. Rev. 2004, 33, 501-513.

[19] D. Gatteschi, A. L. Barra, A. Caneschi, A. Cornia, R. Sessoli, L. Sorace, Coord. Chem. Rev. 2006, 250, 1514-1529.

[20] N. J. Turro, M. H. Kleinman, E. Karatekin, Angew. Chem. 2000, 112, 4608-4634; Angew. Chem. Int. Ed. 2000, 39, 4436-4461.

[21] E. A. Juban, A. L. Smeigh, J. E. Monat, J. K. McCusker, Coord. Chem. Rev. 2006, 250, 1783-1791.

[22] W. R. Browne, J. J. McGarvey, Coord. Chem. Rev. 2006, 250, 16961709 .

[23] M. Ruben, J.-M. Lehn, P. Müller, Chem. Soc. Rev. 2006, 35, 10561067.

[24] J.-P. Cleuziou, W. Wernsdorfer, V. Bouchiat, T. Ondarçuhu, M. Monthioux, Nature Nanotech. 2006, 1, 53-59.

[25] See for instance: a) T. J. Martinez, Acc. Chem. Res. 2006, 39, 119126; b) A. Dreuw, M. Head-Gordon, Chem. Rev. 2005, 105, 40094037.

[26] Organic molecular protopypes: a) S. Nakatsuji, Chem. Soc. Rev. 2004, 33, 348-353, and references therein; b) T. Sugawara, Mol.
Cryst. Liq. Cryst. 1999, 334, 257-273; inorganic molecular prototypes: c) A. Dei, Angew. Chem. 2005, 117, 1184-1187; Angew. Chem. Int. Ed. 2005, 44, 1160-1163, and references therein.

[27] Equally, one might consider opposite transformation even though no example are described in the litterature as far as we know.

[28] Work in progress.

[29] a) G. Rombaut, M. Verelst, S. Golhen, L. Ouahab, C. Mathonière, O. Kahn, Inorg. Chem. 2001, 40, 1151-1159; b) S. Ohkoshi, N. Machida, Z. J. Zhong, K. Hashimoto, Synth. Met. 2001, 122, 523-527.

[30] a) L. Franco, M. Mazzoni, C. Corvaja, V. P. Gubskaya, L. S. Berezhnaya, I. A. Nuretdinov, Chem. Commun. 2005, 2128-2130; b) F. Conti, C. Corvaja, A. Toffoletti, N. Mizuochi, Y. Ohba, S. Yamauchi, M. Maggini, J. Phys. Chem. A 2000, 104, 4962-4967; c) N.; Mizuochi, Y. Ohba, S. Yamauchi, J. Phys. Chem. A 1999, 103, 7749-7750; d) N. Mizuochi, Y. Ohba, S. Yamauchi, J. Phys. Chem. A 1997, 101, 5966-5968; e) C. Corvaja, M.; Maggini, M. Prato, G. Scorrano, M. Venzin, J. Am. Chem. Soc. 1995, 117, 8857-8858.

[31] a) K. Ishii, J. Fujisawa, A. Adachi, S. Yamauchi, N. Kobayashi, J. Am. Chem. Soc. 1998, 120, 3152-3158; b) K. Ishii, J. Fujisawa, Y. Ohba, S. Yamauchi, J. Am. Chem. Soc. 1996, 118, 13079-13080.

[32] a) Y. Teki, T. Toichi, S. Nakajima, Chem. Eur. J. 2006, 12, 23292336; b) T. Toichi, Y. Teki, Polyhedron 2005, 24, 2337-2340; c) Y. Teki, Polyhedron 2005, 24, 2299-2308; d) Y. Teki, Polyhedron 2005, 24, 2185-2188; e) Y. Teki, S. Nakajima, Chem. Lett. 2004, 33, $1500-$ 1501; f) Y. Teki, M. Kimura, S. Narimatsu, K. Ohara, K. Mukai, Bull. Chem. Soc. Jpn. 2004, 77, 95-99; g) Y. Teki, M. Nakatsuji, Y. Miura, Mol. Phys. 2002, 100, 1385-1394; h) Y. Teki, S. Miyamoto, M. Nakatsuji, Y. Miura, J. Am. Chem. Soc. 2001, 123, 294-305; i) Y. Teki, Polyhedron 2001, 20, 1163-1168; j) Y. Teki, S. Miyamoto, K. Iimura, M. Nakatsuji, Y. Miura, J. Am. Chem. Soc. 2000, 122, $984-$ 985.

[33] a) N. Roques, P. Gerbier, Y. Teki, S. Choua, P. Lesniakovà, J.-P. Sutter, P. Guionneau, C. Guérin, New J. Chem. 2006, 30, 1319-1326; b) N. Roques, P. Gerbier, U. Schatzschneider, J.-P. Sutter, P. Guionneau, J. Vidal-Gancedo, J. Veciana, E. Rentschler, C. Guérin, Chem. Eur. J. 2006, 12, 5547-5562; c) N. Roques, P. Gerbier, S. Nakajima, Y. Teki, C. Guérin, J. Phys. Chem. Solids 2004, 65, 759-762.

[34] B. D. Koivisto, R. G. Hicks, Coord. Chem. Rev. 2005, 249, $2612-$ 2630.

[35] D. Luneau, P. Rey, Coord. Chem. Rev. 2005, 249, 2591-2611.

[36] H.-D. Becker, Chem. Rev. 1993, 93, 145-172.

[37] a) P. Huai, Y. Shimoi, S. Abe, Phys. Rev. B 2005, 72, 094413/1-9; b) P. Huai, Y. Shimoi, S. Abe, Synth. Met. 2003, 137, 1255-1256.

[38] Gaussian 03, Revision B.05, M. J. Frisch, G. W. Trucks, H. B. Schlegel, G. E. Scuseria, M. A. Robb, J. R. Cheeseman, J. A. Montgomery, Jr., T. Vreven, K. N. Kudin, J. C. Burant, J. M. Millam, S. S. Iyengar, J. Tomasi, V. Barone, B. Mennucci, M. Cossi, G. Scalmani, N. Rega, G. A. Petersson, H. Nakatsuji, M. Hada, M. Ehara, K. Toyota, R. Fukuda, J. Hasegawa, M. Ishida, T. Nakajima, Y. Honda, O. Kitao, H. Nakai, M. Klene, X. Li, J. E. Knox, H. P. Hratchian, J. B. Cross, V. Bakken, C. Adamo, J. Jaramillo, R. Gomperts, R. E. Stratmann, O. Yazyev, A. J. Austin, R. Cammi, C. Pomelli, J. W. Ochterski, P. Y. Ayala, K. Morokuma, G. A. Voth, P. Salvador, J. J. Dannenberg, V. G. Zakrzewski, S. Dapprich, A. D. Daniels, M. C. Strain, O. Farkas, D. K. Malick, A. D. Rabuck, K. Raghavachari, J. B. Foresman, J. V. Ortiz, Q. Cui, A. G. Baboul, S. Clifford, J. Cioslowski, B. B. Stefanov, G. Liu, A. Liashenko, P. Piskorz, I. Komaromi, R. L. Martin, D. J. Fox, T. Keith, M. A. Al-Laham, C. Y. Peng, A. Nanayakkara, M. Challacombe, P. M. W. Gill, B. Johnson, W. Chen, M. W. Wong, C. Gonzalez, J. A. Pople, Gaussian, Inc., Wallingford CT, 2004.

[39] C. Adamo, V. Barone, J. Chem. Phys. 1999, 110, 6158-6170.

[40] J. P. Perdew, K. Burke, M. Ernzerhof, Phys. Rev. Lett. 1996, 77, 3865-3868.

[41] T. H. Dunning Jr., P. J. Hay, in Modern Theoretical Chemistry (Ed.: H. F. Schaefer, III), Plenum, New York, 1976, pp. 1-28.

[42] W. J. Hehre, R. Ditchfield, J. A. Pople, J. Chem. Phys. 1972, 56, 2257-2261.

[43] W. Heisenberg, Z. Phys. 1928, 49, 619-636. 
[44] P. A. M. Dirac, Proc. R. Soc. London Ser. A 1929, 123, 714-733.

[45] J. H. Van Vleck, The Theory of Electric and Magnetic Susceptibilities, Oxford University Press, New York, 1932.

[46] a) L. Noodleman, J. G. Norman, J. Chem. Phys. 1979, 70, 4903 4906; b) L. Noodleman, J. Chem. Phys. 1981, 74, 5737-5743; c) J. G. Norman, P. B. Ryan, L. Noodleman, J. Am. Chem. Soc. 1980, 102, 4279-4282.

[47] A. A. Ovchinnikov, J. K. Labanowski, Phys. Rev. A 1996, 53, 39463952.

[48] I. Ciofini, C. A. Daul, Coord. Chem. Rev. 2003, 238-239, 187-209.

[49] W. Weltner, Magnetic Atoms and Molecules, Dover, New York 1989.

[50] F. Neese, ORCA: An ab-initio, Density Functional and Semi-empirical electronic structure Package, Version 2.4 Revision 41, MaxPlanck Institut für Bioanorganische Chemie, Mülheim (Germany), 2004.

[51] A. J. Stone, Proc. R. Soc. London Ser. A 1963, 271, 424-434.

[52] S. Koseki, M. W. Schmidt, M. S. Gordon, J. Phys. Chem. 1992, 96, 10768-10772.

[53] O. Loboda, B. Minaev, O. Vahtras, B. Schimmelpfennig, H. Ågren, K. Ruud, D. Jonsson, Chem. Phys. 2003, 286, 127-137.

[54] S. Sinnecker, F. Neese, J. Phys. Chem. A 2006, 110, 12267-12275.

[55] a) P. P. Lainé, F. Bedioui, F. Loiseau, C. Chiorboli, S. Campagna, J. Am. Chem. Soc. 2006, 128, 7510-7521; b) P. P. Lainé, F. Loiseau, S. Campagna, I. Ciofini, C. Adamo, Inorg. Chem. 2006, 45, 5538-5551; c) P. P. Lainé, I. Ciofini, P. Ochsenbein, E. Amouyal, C. Adamo, F. Bedioui, Chem. Eur. J. 2005, 11, 3711-3727; d) I. Ciofini, P. P. Lainé, F. Bedioui, C. Adamo, J. Am. Chem. Soc. 2004, 126, 10763-10777.

[56] E. A. Weiss, M. J. Tauber, R. F. Kelley, M. J. Ahrens, M. A. Ratner, M. R. Wasielewski, J. Am. Chem. Soc. 2005, 127, 11842-11850.

[57] M. Cotlet, S. Masuo, G. Luo, J. Hofkens, M. van der Auweraer, J. Verhoeven, K. Müllen, X. S. Xie, F. De Schryver, Proc. Natl. Acad. Sci. USA 2004, 101, 14343-14348.

[58] O. Castell, R. Caballol, R. Subra, A. Grand, J. Phys. Chem. 1995, 99 , $154-157$.

[59] V. Barone, A. Bencini, A. di Matteo, J. Am. Chem. Soc. 1997, 119, $10831-10837$.

[60] a) R. G. Hicks, M. T. Lemaire, L. Öhrström, J. F. Richardson, L. K. Thompson, Z. Xu, J. Am. Chem. Soc. 2001, 123, 7154-7159; b) D. J. R. Brook, H. R. Fox, V. Lynch, M. A. Fox, J. Phys. Chem. 1996, 100, 2066-2071; c) F. A. Neugebauer, H. Fischer, C. Krieger, J. Chem. Soc. Perkin Trans. 2 1993, 3, 535-544.

[61] V. Barone, A. Bencini, I. Ciofini, C. A. Daul, J. Phys. Chem. A 1999, $103,4275-4282$.

[62] The term "planarization" specifically refers to a relaxation process of the molecular structure towards planarity on going from the ground state to the lowest photoexcited state regardless of its spin multiplicity.

[63] a) A. Karpfen, C. H. Choi, M. Kertesz, J. Phys. Chem. A 1997, 101, $7426-7433$; b) At the PBE0/LANL2 level of theory, the barrier for rigid rotation of biphenyl has been computed to be $3.0 \mathrm{kcal} \mathrm{mol}^{-1}$.

[64] When considering the cis isomer for Td1, an exchange-coupling constant of $-5.4 \mathrm{~K}$ was computed, in better agreement with experimental issues.

[65] A. Bencini, F. Totti, C. A. Daul, K. Doclo, V. Barone, Inorg. Chem. 1997, 36, 5022-5030.

[66] a) Md. E. Ali, S. N. Datta, J. Phys. Chem. A 2006, 110, 2776-2784; b) S. Vyas, Md. E. Ali, E. Hossain, S. Patwardhan, S. N. Datta, J. Phys. Chem. A 2006, 110, 4213-4215; c) Md. E. Ali, S. Vyas, S. N. Datta, J. Phys. Chem. A 2005, 109, 6272-6278.

[67] L. Catala, J. Le Moigne, N. Gruber, J. J. Novoa, P. Rabu, E. Belorizky, P. Turek, Chem. Eur. J. 2005, 11, 2440-2454.
[68] W. T. Borden, E. R. Davidson, J. Am. Chem. Soc. 1977, 99, 45874594.

[69] I. Ciofini, C. Adamo, V. Barone, J. Chem. Phys. 2004, 121, 67106718.

[70] F. Neese, J. Chem. Phys. 2001, 115, 11080-11096.

[71] I. Ciofini, C. Adamo, V. Barone, G. Berthier, A. Rassat, Chem. Phys. 2005, 309, 133-141.

[72] Enhanced intersystem crossing experimentally evidenced has been demonstrated to favor ferromagnetic coupling and is therefore described as selective; see reference [32].

[73] In spite of noticeable differences between the herein derived GS/ES functional models and those used in the literature, modeling of spectroscopic outcomes (especially TR-ESR data) performed by Teki has not been affected. Indeed, throughout his work, parameter quantities related to [ph-An-ph] spin coupler were systematically approximated by using those attached to [An] (and $\left[{ }^{3} \mathrm{An}\right]$ ) to compute the spin Hamiltonian.

[74] $\theta_{2}$ angle about the B-SC connection is systematically smaller than $3^{\circ}$, already corresponding to the optimal situation for mediation of supexchange coupling, and is therefore non-determining for overall magnetic properties.

[75] Beyond steric drawbacks, it is also worth noting that linking the IN radical at the $\mathrm{C}^{\mathrm{ph}}$ position (naturally showing very small ASP) of the phenyl connector results in a destructive interference pattern between the spin densities attached to *SC and SC over the B domain, further weakening local ASP and consequently the strength of superexchange coupling (in this case expected to be antiferro in the excited state).$^{[32 a, e]}$

[76] Case of the representative 9,10-diphenyl-anthracene reference: for the triplet state of isolated anthracene, ASPs located at key connection sites are the following : $0.75 \mathrm{e}^{-}$electron at $\mathrm{C} 9^{\mathrm{An}}$ and $\mathrm{C} 10^{\mathrm{An}}$ positions, $0.25 \mathrm{e}^{-}$at $\mathrm{C} 1^{\mathrm{An}}$ and $0.08 \mathrm{e}^{-}$at the $\mathrm{C} 2^{\mathrm{An}}$ site.

[77] T. Ishida, H. Iwamura, J. Am. Chem. Soc. 1991, 113, 4238-4241.

[78] This work.

[79] R. A. Pascal Jr. , Chem. Rev. 2006, 106, 4809-4819.

[80] Even if exchange couplings of dangling radicals with central anthracene are of different magnitudes, they are anticipated to overcome direct deleterious and through-space (weaker) dipolar interactions between neighboring SCs.

[81] Unpublished results.

[82] Various approaches towards (supra)molecular spintronics exist, see for instance: a) M. Ruben, E. Breuning, J.-M. Lehn, V. Ksenofontov, F. Renz, P. Gütlich, G. B. M. Vaughan, Chem. Eur. J. 2003, 9, 44224429; b) A. Dei, D. Gatteschi, C. Sangregorio, L. Sorace, Acc. Chem. Res. 2004, 37, 827-835; c) E. T. Chernick, Q. Mi, R. F. Kelley, E. A. Weiss, B. A. Jones, T. J. Marks, M. A. Ratner, M. R. Wasielewski, J. Am. Chem. Soc. 2006, 128, 4356-4364, and references therein; d) R. Liu, S.-H. Ke, H. U. Baranger, W. Yang, Nano Lett. 2005, 5, 1959-1962; e) S. Sanvito, A. R. Rocha, J. Comput. Theor. Nanosci. 2006, 3, 624-642, and references therein; f) F Meier, V. Cerletti, O. Gywat, D. Loss, D. D. Awschalom, Phys. Rev. $B$ 2004, 69, 195315/1-195315/12; g) V. N. Prigodin, N. P. Rju, K. I. Pokhodnya, J. S. Miller, A. J. Epstein, Adv. Mater. 2002, 14, 12301233.

[83] a) M. Johnson, J. Phys. Chem. B 2005, 109, 14278-14291; b) M. E. Flatté, Nature 2004, 427, 21-22; c) Y. Kato, R. C. Myers, A. C. Gossard, D. D. Awschalom, Nature 2004, 427, 50-53; d) S. A. Wolf, D. D. Awschalom, R. A. Buhrman, J. M. Daughton, S. von Molnar, M. L. Roukes, A. Y. Chtchelkanova, D. M. Treger, Science 2001, 294, 1488-1495. 
Intram olecular Spin A Jignm ent in Photo-M agnetic M olecular D evices: a Theoretical Study

Tlaria C iofini,* Philippe P. Lainé,* M arta Zam boni, C laude A .D aul, V alérie M arvaud, and Carlo A dam o 
Table SI-I. Structural parameters computed for imino-nitroxide (IN) derivatives Tdl and DdI and the ground state (Td1, DdI) and in the excited state (Td1*, Dd1*) as a function of the overall spin state. Available X-ray data for similar systems are given for comparison purposes. Distances in $\AA$, angles in degrees. For labeling scheme refer to Figure la.

\begin{tabular}{|c|c|c|c|c|c|c|c|}
\hline & & Td1 & Td1 & Td1* & Dd1 & Dd1* & IN $\left(\exp \cdot{ }^{[a]}\right)$ \\
\hline & BS & $\left(m_{S}=0\right)$ & $S=1$ & $S=2$ & $S=1 / 2$ & $S=3 / 2$ & $S=1 / 2$ \\
\hline $\mathrm{dC}_{9}{ }^{\mathrm{An}} \mathrm{C}_{1}{ }^{\mathrm{Ph}}$ & & 1.479 & 1.479 & 1.4790 & 1.492 & 1.476 & \\
\hline $\mathrm{dC}_{4}{ }^{\mathrm{Ph}} \mathrm{C}_{2}{ }^{\mathrm{IN}}$ & & 1.471 & 1.471 & 1.4677 & 1.471 & 1.467 & $1.472 / 1.476$ \\
\hline $\mathrm{dC}_{2}{ }^{\mathrm{IN}} \mathrm{N}_{1}$ & & 1.419 & 1.419 & 1.418 & 1.419 & 1.418 & $1.380 / 1.369$ \\
\hline $\mathrm{dC}_{2}{ }^{\mathrm{IN}} \mathrm{N}_{3}$ & & 1.311 & 1.311 & 1.313 & 1.311 & 1.313 & $1.279 / 1.243$ \\
\hline $\mathrm{dC}_{4}{ }^{\mathrm{IN}} \mathrm{N}_{3}$ & & 1.486 & 1.486 & 1.486 & 1.486 & 1.486 & $1.481 / 1.503$ \\
\hline $\mathrm{dC}_{5}{ }^{\mathrm{IN}} \mathrm{N}_{1}$ & & 1.484 & 1.484 & 1.484 & 1.484 & 1.484 & $1.483 / 1.477$ \\
\hline $\mathrm{dN}_{1} \mathrm{O}$ & & 1.305 & 1.305 & 1.305 & 1.305 & 1.305 & $1.268 / 1.270$ \\
\hline $\mathrm{a}\left(\mathrm{N}_{1} \mathrm{C}_{2}{ }^{\mathrm{IN}} \mathrm{N}_{3}\right)$ & & 112.4 & 112.4 & 112.4 & 112.4 & 112.4 & $112.2 / 110.7$ \\
\hline 1 & & 73.5 & 73.5 & 59.1 & 71.2 & 56.4 & \\
\hline 2 & & 1.1 & 1.1 & 1.2 & 2.4 & 1.1 & $7.0 / 2.2$ \\
\hline
\end{tabular}

[a] From X-ray structure of 1,1-dimethyl-2,5-bis-(iminonitroxyl-phenyl) 3,4-diphenylsilole (TPSIN). ${ }^{[33 a]}$

Table SI-II. Structural parameters computed for oxo-verdazyl (OV) derivatives $T d 2$ and $D d 2$ in the ground state (Td2, Dd2) and in the excited state $(\mathrm{Td} 2 *, \mathrm{Dd} 2 *)$ as a function of the overall spin state. Available $\mathrm{X}$-ray data for similar systems are given for comparison purposes. Distances in $\AA$, angles in degrees. For labeling scheme refer to Figure 1a. [a]

\begin{tabular}{cccccccccc}
\hline & $\mathrm{Td} 2$ & $\mathrm{Td} 2$ & $\mathrm{Td} 2$-notB & $\mathrm{Td2}$ * & $\mathrm{Td2}$-notB & $\mathrm{Dd} 2$ & $\mathrm{Dd2}$ & $\mathrm{OV}$ (exp.) \\
& $\mathrm{BS}\left(\mathrm{m}_{\mathrm{S}}=0\right)$ & $\mathrm{S}=1$ & $\mathrm{~S}=1$ & $\mathrm{~S}=2$ & $\mathrm{~S}=2$ & $\mathrm{~S}=1 / 2$ & $\mathrm{~S}=3 / 2$ & $\mathrm{~S}=1 / 2$ \\
\hline $\mathrm{dC}_{9}{ }^{\mathrm{An}} \mathrm{C}_{1}{ }^{\mathrm{Ph}}$ & 1.493 & 1.492 & 1.493 & 1.478 & 1.479 & 1.493 & 1.476 & &
\end{tabular}




$\begin{array}{lcccccccc}\mathrm{dC}_{4}{ }^{\mathrm{Ph}} \mathrm{C}_{1}{ }^{\mathrm{ov}} & 1.477 & 1.477 & 1.477 & 1.473 & 1.474 & 1.477 & 1.473 \\ \mathrm{dC}_{1}{ }^{\mathrm{ov}} \mathrm{N}_{2} & 1.354 & 1.354 & 1.354 & 1.355 & 1.355 & 1.354 & 1.355 & 1.321(4) / 1.323(4){ }^{[\mathrm{c}]} \\ \mathrm{dN}_{2} \mathrm{~N}_{3} & 1.379 & 1.380 & 1.379 & 1.379 & 1.379 & 1.379 & 1.379 & 1.365(3) / 1.358(3){ }^{[\mathrm{c}]} \\ \mathrm{dC}_{2}{ }^{\mathrm{ov}} \mathrm{N}_{3} & 1.395 & 1.393 & 1.395 & 1.395 & 1.394 & 1.395 & 1.395 & 1.379(4) / 1.367(4){ }^{[\mathrm{c}]} \\ \mathrm{dC}_{4}{ }^{\mathrm{ov}} \mathrm{O} & 1.248 & 1.248 & 1.248 & 1.248 & 1.248 & 1.248 & 1.248 & 1.217(3)^{[\mathrm{c}]} \\ \mathrm{dN}_{3} \mathrm{R} & 1.459 & 1.460 & 1.460 & 1.459 & 1.459 & 1.459 & 1.459 & 1.460(4) / 1.454(4){ }^{[\mathrm{c}]} \\ \mathrm{a}\left(\mathrm{N}_{6} \mathrm{C}_{1}{ }^{\mathrm{ov}} \mathrm{N}_{2}\right) & 125.6 & 125.7 & 125.6 & 125.5 & 125.6 & 125.6 & 125.5 \\ 1 & 76.0 & 71.1 & 75.7 & 58.3 & 59.8 & 72.7 & 55.9 \\ 1 & 0.8 & 0.6 & 0.9 & 0.6 & 0.8 & 0.4 & 0.9\end{array}$

[a] "notB" name extension refers to molecules deprived of t-butyl group on their An core. [b] X-ray structure of 1,3,5-triphenyl-6-oxoverdazyl from ref. [60c]. [c] X-ray structure of 1,5-dimethyl-3-(2-pyridyl)-6-oxoverdazyl radical from ref. [60a].

Table SI-III Structural parameters computed for imino-nitroxide derivatives Td3/4 and Dd3/4 in the ground state (Td3, Dd3, Td4 and Dd4) and in the excited state (Td3*, Dd3*, Td4* and Dd4*) as a function of the overall spin state. Available X-ray data for similar systems are given for comparison purposes. Distances in $\AA$, angles in degrees. For labeling scheme refer to Figure $1 \mathrm{~b}$.

\begin{tabular}{|c|c|c|c|c|c|c|}
\hline & $\mathrm{Td}^{\lfloor\mathrm{a}\rfloor}$ & $\operatorname{Td} 3^{[a]}$ & $\mathrm{Td} 3 *^{[\mathrm{a}]}$ & Dd3 & Dd3* & IN $\left(\exp .^{[b]}\right)$ \\
\hline & BS $\left(m_{S}=0\right)$ & $S=1$ & $S=2$ & $S=1 / 2$ & $S=3 / 2$ & $S=1 / 2$ \\
\hline $\mathrm{dC}_{9}{ }^{\mathrm{An}} \mathrm{C}_{1}{ }^{\mathrm{Pn}}$ & $1.493 / 1.487$ & $1.493 / 1.487$ & $1.477 / 1.479$ & 1.487 & 1.478 & \\
\hline $\mathrm{dC}_{4}{ }^{\mathrm{Ph}} \mathrm{C}_{2}{ }^{\mathrm{IN}}$ & $1.471 / 1.470$ & $1.471 / 1.470$ & $1.467 / 1.468$ & 1.470 & 1.468 & $1.472 / 1.476$ \\
\hline $\mathrm{dC}_{2}{ }^{\mathrm{IN}} \mathrm{N}_{1}$ & $1.419 / 1.419$ & $1.419 / 1.419$ & $1.418 / 1.419$ & 1.419 & 1.419 & $1.380 / 1.369$ \\
\hline $\mathrm{dC}_{2}{ }^{\mathrm{IN}} \mathrm{N}_{3}$ & $1.311 / 1.311$ & $1.311 / 1.311$ & $1.313 / 1.313$ & 1.311 & 1.313 & $1.279 / 1.243$ \\
\hline $\mathrm{dC}_{4}{ }^{\mathrm{IN}} \mathrm{N}_{3}$ & $1.486 / 1.486$ & $1.486 / 1.486$ & $1.486 / 1.486$ & 1.486 & 1.486 & $1.481 / 1.503$ \\
\hline $\mathrm{dC}_{5}{ }^{\mathrm{IN}} \mathrm{N}_{1}$ & $1.484 / 1.484$ & $1.484 / 1.484$ & $1.484 / 1.484$ & 1.484 & 1.484 & $1.483 / 1.477$ \\
\hline $\mathrm{dN}_{1} \mathrm{O}$ & $1.305 / 1.305$ & $1.305 / 1.305$ & $1.305 / 1.305$ & 1.305 & 1.305 & $1.268 / 1.270$ \\
\hline $\mathrm{a}\left(\mathrm{N}_{1} \mathrm{C}_{2}{ }^{\mathrm{IN}} \mathrm{N}_{3}\right)$ & $112.4 / 112.4$ & $112.4 / 112.4$ & $112.4 / 112.4$ & 112.4 & 112.4 & $112.2 / 110.7$ \\
\hline 1 & $-72.2 /-51.8$ & $-72.2 /-51.8$ & $-56.3 /-44.6$ & -51.8 & -44.4 & \\
\hline 2 & $0.7 / 1.3$ & $0.7 / 1.3$ & $1.6 /-1.0$ & 1.9 & 1.7 & $2.2 / 7.0$ \\
\hline
\end{tabular}




\begin{tabular}{|c|c|c|c|c|c|c|}
\hline & $\mathrm{Td} 4^{[\mathrm{C}]}$ & $\mathrm{Td} 4^{[\mathrm{lc}]}$ & $\mathrm{Td} 4 *^{[\mathrm{C}]}$ & Dd4 & Dd4 * & IN $\left(\exp .^{[D]}\right)$ \\
\hline & BS, $\quad\left(m_{S}=0\right)$ & $S=1$ & $S=2$ & $S=1 / 2$ & $S=3 / 2$ & $S=1 / 2$ \\
\hline $\mathrm{dC}_{9}{ }^{\mathrm{An}} \mathrm{C}_{1}{ }^{\mathrm{Ph}}$ & $1.471 / 1.469$ & $1.471 / 1.469$ & $1.478 / 1.475$ & 1.481 & 1.473 & \\
\hline $\mathrm{dC}_{4}{ }^{\mathrm{Ph}} \mathrm{C}_{2}{ }^{\mathrm{IN}}$ & $1.471 / 1.469$ & $1.471 / 1.469$ & $1.468 / 1.467$ & 1.469 & 1.467 & $1.472 / 1.476$ \\
\hline $\mathrm{dC}_{2}{ }^{\mathrm{IN}} \mathrm{N}_{1}$ & $1.419 / 1.419$ & $1.419 / 1.419$ & $1.418 / 1.419$ & 1.419 & 1.419 & $1.380 / 1.369$ \\
\hline $\mathrm{dC}_{2}{ }^{\mathrm{IN}} \mathrm{N}_{3}$ & $1.311 / 1.311$ & $1.311 / 1.311$ & $1.313 / 1.313$ & 1.311 & 1.313 & $1.279 / 1.243$ \\
\hline $\mathrm{dC}_{4}{ }^{\mathrm{IN}} \mathrm{N}_{3}$ & $1.486 / 1.486$ & $1.486 / 1.486$ & $1.485 / 1.486$ & 1.486 & 1.486 & $1.481 / 1.503$ \\
\hline $\mathrm{dC}_{5}{ }^{\mathrm{IN}} \mathrm{N}_{1}$ & $1.484 / 1.484$ & $1.484 / 1.484$ & $1.484 / 1.484$ & 1.484 & 1.484 & $1.483 / 1.477$ \\
\hline $\mathrm{dN}_{1} \mathrm{O}$ & $1.305 / 1.305$ & $1.305 / 1.305$ & $1.305 / 1.305$ & 1.305 & 1.305 & $1.268 / 1.270$ \\
\hline $\mathrm{a}\left(\mathrm{N}_{1} \mathrm{C}_{2}{ }^{\mathrm{IN}} \mathrm{N}_{3}\right)$ & $112.4 / 112.4$ & $112.4 / 112.4$ & $112.4 / 112.4$ & 112.4 & 112.4 & $112.2 / 110.7$ \\
\hline 1 & $-72.2 / 33.4$ & $-72.2 / 33.4$ & $-57.3 / 28.6$ & 33.3 & 27.8 & \\
\hline 2 & $1.1 /-0.5$ & $1.1 /-0.5$ & $1.1 /-1.2$ & -1.2 & -0.8 & $2.2 / 7.0$ \\
\hline
\end{tabular}

[a] The first value corresponds to the iminonitroxide linked at position $\mathrm{C}_{10}{ }^{\mathrm{An}}$; the second to that at position $\mathrm{C}_{1}{ }^{\mathrm{An}}$. [b] From X-ray structure of 1,1dimethyl-2,5-bis-(iminonitroxyl-phenyl)-3,4-diphenylsilole (TPSIN). [33a] [C] The first value corresponds to the iminonitroxide linked at position $\mathrm{C}_{10}{ }^{\mathrm{An}}$, the second to that at position $\mathrm{C}_{2}{ }^{\mathrm{An}}$. 


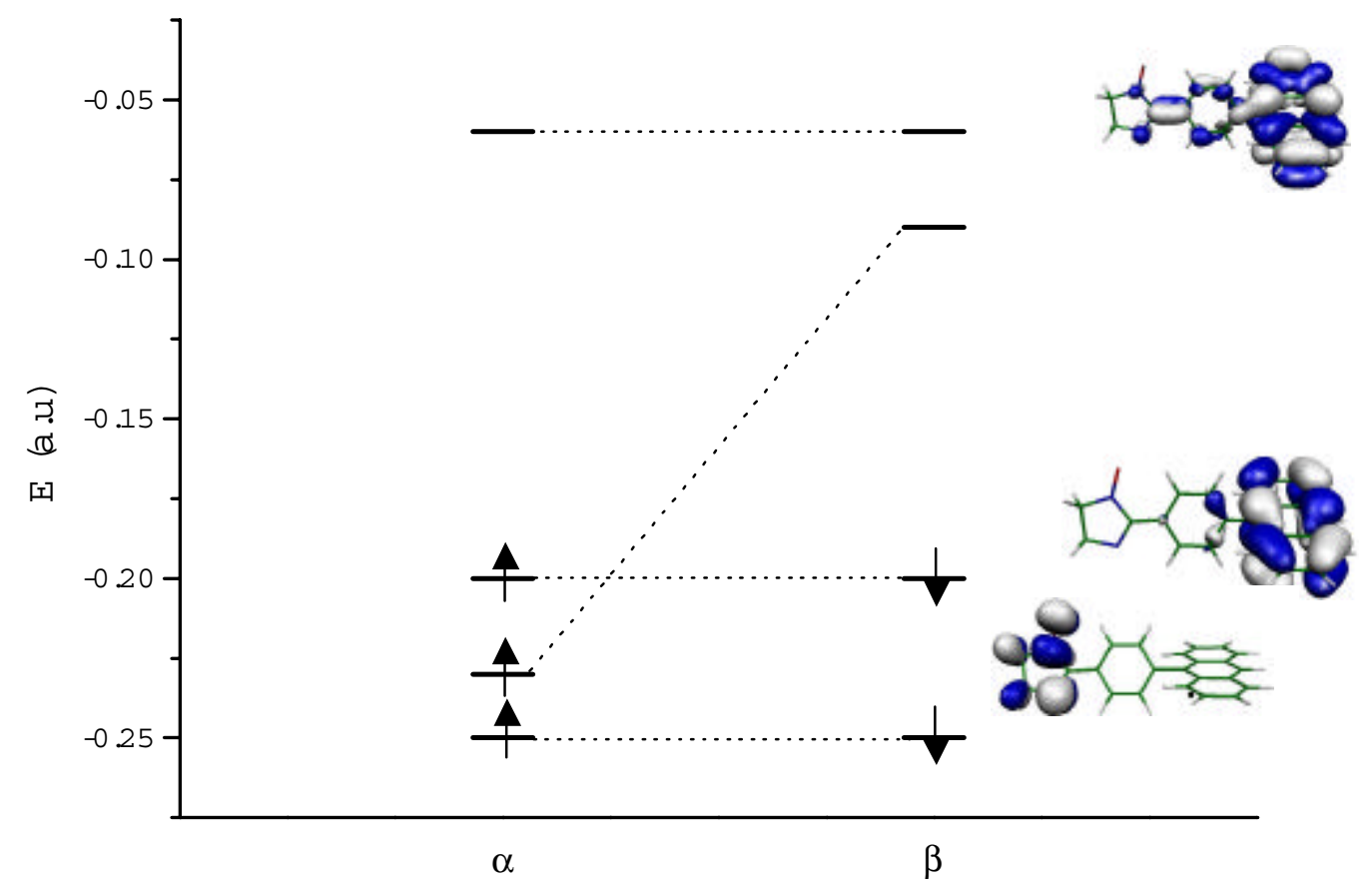

Figure SI-1. MO scheme computed for Dd1. Shadow: MOs not involved in the photomagnetic behavior of the system. Contour value 0.025 a.u.

The energies of the highest occupied (HOMO) and lowest unoccupied (LUMO) MOs of the representative dyad Dd1, for instance, are reported in Figure SI-1 together with an isovalue representation of the orbitals involved in the magnetic and photophysical processes. Since all calculations were performed using an unrestricted formalism, the energies 
of the alpha and the corresponding beta orbitals are not necessarily degenerate (a dashed line is drawn to connect each alpha to its beta counterpart). The HOMOs (alpha and beta) are localized on the An units while the Singly Occupied MO (lower in energy by ca. $0.8 \mathrm{eV}$ ) is exclusively localized on the IN moiety. No sizable coupling between the An, ph and IN units is therefore computed in the ground state. Upon excitation, one electron (beta) is promoted from the HOMO to the LUMO (alpha). This orbital is almost completely localized on the An, thus confirming that the transition corresponds to the formation of a locally excited state ${ }^{3} \mathrm{An}$. Indeed, only a (very) small contribution located on the phenyl B subunit and on the IN can be found. Interestingly, a bonding interaction between both $\mathrm{C}_{9}{ }^{\mathrm{An}}-\mathrm{C}_{1}{ }^{\mathrm{Ph}}$ and $\mathrm{C}_{4}{ }^{\mathrm{Ph}}-\mathrm{C}_{1}{ }^{\mathrm{IN}}$ is however computed. This interaction is responsible for the above-mentioned planarization about 1 (and to a lesser extent about 2) found to occur upon photon excitation (Tables 1 and 2 ).
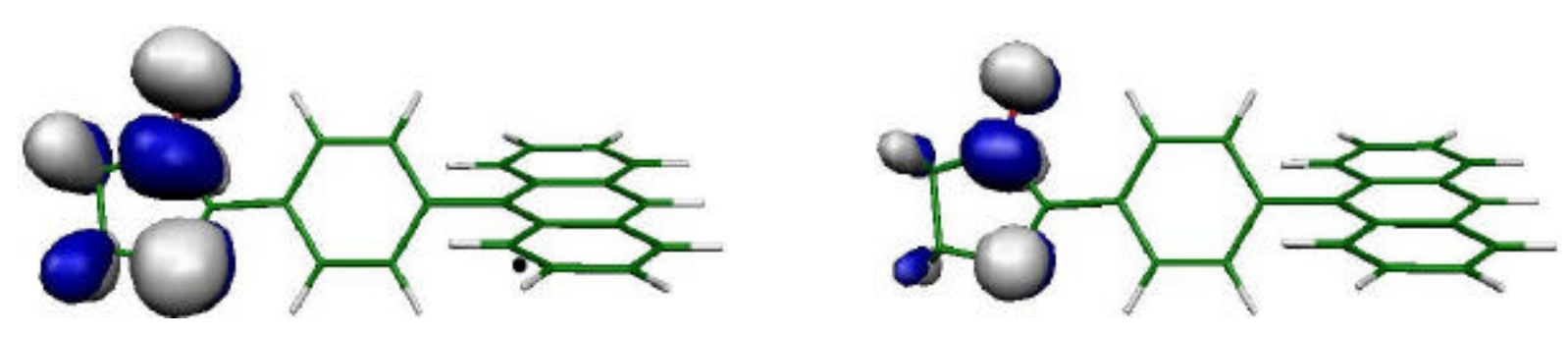

Figure SI-2. SOMO of Dd1. Contour value $0.025 \mathrm{a} . \mathrm{u}$. (left) and 0.05 a.u. (right). 

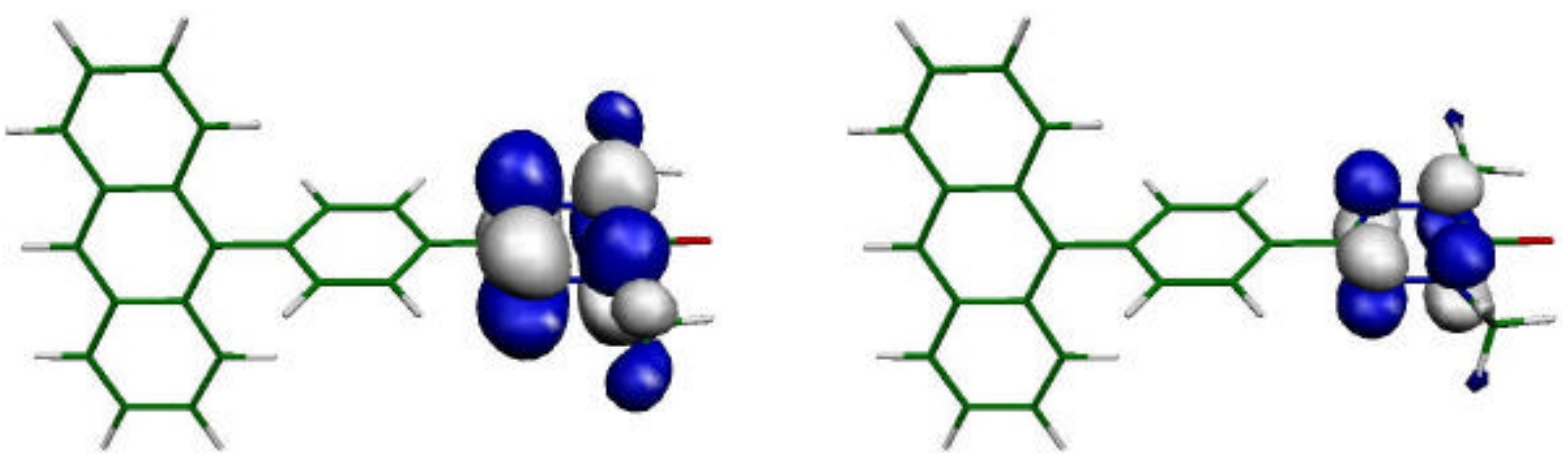

Figure SI-3. SOMO of Dd2. Contour value 0.025 a.u. (left) and 0.05 a.u. (right).
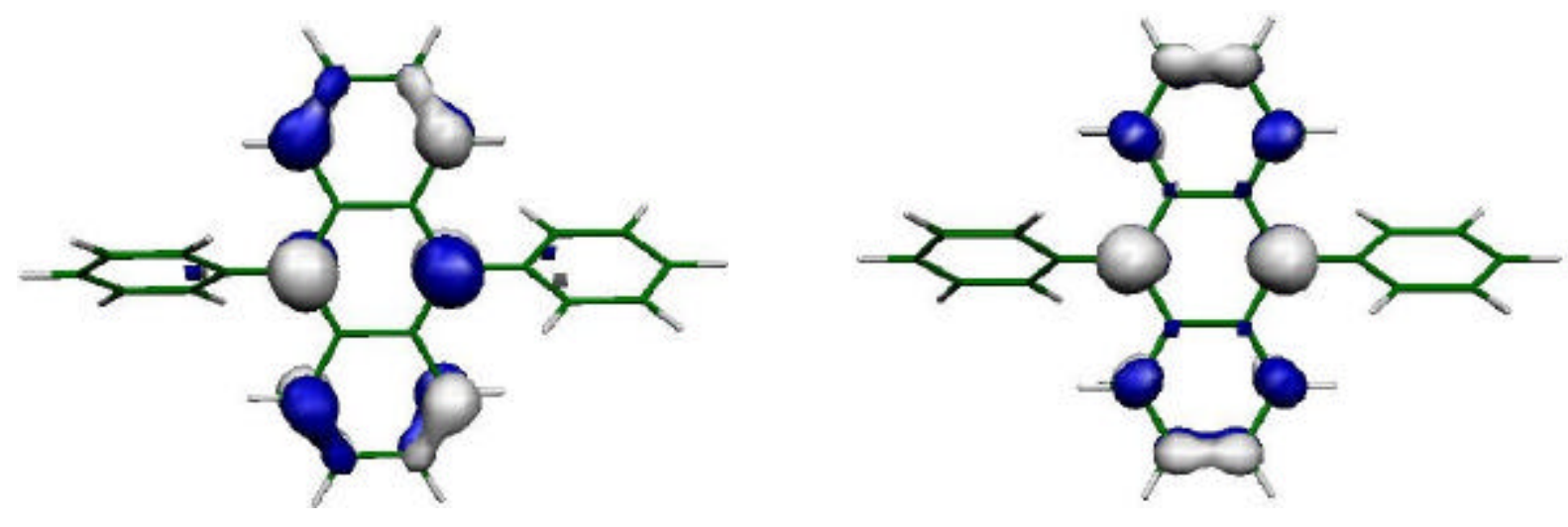

Figure SI-4. SOMOs of ${ }^{3}[\mathrm{ph}-\mathrm{An}-\mathrm{ph}]$. Contour value $0.05 \mathrm{a} . \mathrm{u}$. 